\title{
An Overview of the HomePlug AV2 Technology
}

\author{
Larry Yonge, ${ }^{1}$ Jose Abad, ${ }^{2}$ Kaywan Afkhamie, ${ }^{1}$ \\ Lorenzo Guerrieri, ${ }^{3}$ Srinivas Katar, ${ }^{1}$ Hidayat Lioe, ${ }^{4}$ Pascal Pagani, ${ }^{5}$ \\ Raffaele Riva, ${ }^{6}$ Daniel M. Schneider, ${ }^{7}$ and Andreas Schwager ${ }^{7}$ \\ ${ }^{1}$ Qualcomm Atheros, 203 East Silver Springs Boulevard, Ocala, FL 34470, USA \\ ${ }^{2}$ Broadcom Corporation, Llacuna 56-70, 8th Floor, Building A, 08005 Barcelona, Spain \\ ${ }^{3}$ DORA S.p.A., STMicroelectronics Group, Via Lavoratori Vittime del Col Du Mont 28, 11100 Aosta, Italy \\ ${ }^{4}$ Marvell Semiconductors, 5488 Marvell lane, Santa Clara, CA 95054, USA \\ ${ }^{5}$ Orange Labs Networks, 2 Avenue Pierre Marzin, 22300 Lannion, France \\ ${ }^{6}$ STMicroelectronics S.r.l., Via Olivetti 2, 20864 Agrate Brianza, Italy \\ ${ }^{7}$ Sony, Hedelfinger Straße 61, 70327 Stuttgart, Germany \\ Correspondence should be addressed to Pascal Pagani; pascal.pagani@ieee.org
}

Received 3 August 2012; Accepted 21 November 2012

Academic Editor: Andrea M. Tonello

Copyright ( $\odot 2013$ Larry Yonge et al. This is an open access article distributed under the Creative Commons Attribution License, which permits unrestricted use, distribution, and reproduction in any medium, provided the original work is properly cited.

HomePlug AV2 is the solution identified by the HomePlug Alliance to achieve the improved data rate performance required by the new generation of multimedia applications without the need to install extra wires. Developed by industry-leading participants in the HomePlug AV Technical Working Group, the HomePlug AV2 technology provides Gigabit-class connection speeds over the existing AC wires within home. It is designed to meet the market demands for the full set of future in-home networking connectivity. Moreover, HomePlug AV2 guarantees backward interoperability with other HomePlug systems. In this paper, the HomePlug AV2 system architecture is introduced and the technical details of the key features at both the PHY and MAC layers are described. The HomePlug AV2 performance is assessed, through simulations reproducing real home scenarios.

\section{Introduction}

The convergence of voice, video, and data within a variety of multifunction devices, along with the evolution of High Definition (HD) and 3-Dimensional (3D) video, are today driving the demands for home connectivity solutions. Home networks are required to support high throughput connectivity guaranteeing at the same time a high level of reliability and coverage (the percentage of links that are able to sustain a given throughput in two-node or multinode networks). Applications such as HD Television (HDTV), Internet Protocol Television (IPTV), interactive gaming, whole-home audio, security monitoring, and Smart Grid management have to be supported by the new home networks.

During the last decade, in-home power line communication (PLC) has received increasing attention from both the industry and research communities. The reuse of existing wires to deploy wide band services is the main source of attractiveness of the in-home PLC technologies. Another major advantage of PLC is the ubiquity of the power lines which can be used to provide whole-home connectivity solutions. However, the power line medium has not been originally designed for data communication; the frequency selectivity of the channel and different types of noise (background noise, impulsive noise, and narrow band interferers) make the power line a very challenging environment and require state of the art design solutions.

In 2000, the HomePlug Alliance [1], an industry-led organization, was formed with the scope to promote power line networking through the adoption of HomePlug specifications. In 2001, the HomePlug Alliance released the HomePlug 1.0.1 specification followed in 2005 by a second release named HomePlug AV [2]. The letters "AV" abbreviate "Audio, Video". HPAV rapidly became the most widespread adopted solution 
for in-home power line communication. To meet the future market needs, in January 2012 the HomePlug Alliance published the HomePlug AV2 specification [3]. HomePlug AV2 enables Gigabit-class connection speeds by leveraging on the existing power line wires while remaining fully interoperable with other technologies for in-home connectivity (HomePlug AV [2], HomePlug Green PHY [4], and IEEE 1901 [5]). The Alliance's AV Technical Working Group (AV TWG) defined new features at both the PHY and MAC layers. These have been introduced in the HomePlug AV2 specification based on extensive field tests conducted in real home scenarios across different countries. The field results obtained by the AV TWG validated the HomePlug AV2 claimed performance both in terms of achievable data rate and coverage.

In this paper, we highlight the key differentiating HomePlug AV2 features compared to the HomePlug AV technology. At the physical (PHY) layer HomePlug AV2 includes the following. (i) Multiple-Input Multiple-Output (MIMO) signaling with Beamforming to offer the benefit of improved coverage throughout the home, especially on highly attenuated channels. MIMO enables HomePlug AV2 devices to transmit on any two-wire pairs within three-wire configurations comprising Line (L), Neutral (N) and Protective Earth (PE) (the coupling is done in the MIMO Analog Front End (AFE) blocks in Figure 1). (ii) Extended Frequency Band up to $86 \mathrm{MHz}$ to increase the throughput especially at the low to mid coverage percentages (part of IFFT/FFT $(1024,8192)$ and Mapper blocks in Figure 1). (iii) Efficient Notching allows transmitters to create extremely sharp frequency notches. If Electromagnetic Compatibility (EMC) regulation will require a fragmented communication spectrum, the throughput loss by excluded frequencies can be minimized (part of Power Allocation and Window and Overlap blocks in Figure 1). (iv) Power back-off to increase the HomePlug AV2 data rate while reducing the electromagnetic emissions (part of Power Allocation blocks and the AFEs in Figure 1). (v) EMC Friendly Power Boost to optimize the transmit power by monitoring the input port reflection coefficient (known as the $S_{11}$ parameter) at the transmitting modem. (vi) Additional PHY Improvements, comprising higher order Quadrature Amplitude Modulation (4096-QAM) (part of Mapper and Demodulator blocks in Figure 1), higher Code Rates (8/9 code rate) (part of Turbo Convolutional Encoder in Figure 1) and smaller guard intervals (part of Cyclic Prefix blocks in Figure 1), to assist better peak data rates. At the Medium Access Control (MAC) layer, HomePlug AV2 includes the following. (i) Power Save mode to improve energy efficiency when the device is in standby. (ii) Short Delimiter to reduce the overhead of the transmission shortening the Preamble and Frame Control symbols. (iii) Delayed Acknowledgements to increase the overall transmission efficiency by reducing the Inter Frame Spacings. (iv) Immediate Repeating to expand the coverage by repeating the signal on paths with better Signal to Noise Ratio (SNR) characteristics.

All the features listed above improve the Quality of Service of AV2 power line modems, by improving coverage and robustness of communication links.

The following sections provide technical details for all the above mentioned features. Moreover, the AV TWG evaluated the performance of the HomePlug AV2 system. The adoption of all the above listed features provides a significant gain of HomePlug AV2 compared to HomePlug AV. The remainder of this paper is organized as follows. Section 2 presents the overall HomePlug AV2 system architecture preceded by a brief overview of the HomePlug AV technology. The new PHY and MAC layer technical features are detailed in Sections 3 and 4, respectively. Section 5 explains how the technology coexistence is dealt with. Section 6 summarizes the performance gain of HomePlug AV2 compared to HomePlug AV and Section 7 concludes this introduction to the HomePlug AV2 technology.

\section{System Architecture}

2.1. A Brief Overview of HomePlug AV. HomePlug AV employs PHY and MAC technology that provides a 200Mbps class power line networking capability. The PHY operates in the frequency range of $2-28 \mathrm{MHz}$, uses windowed Orthogonal Frequency Division Multiplexing (OFDM) and a powerful Turbo Convolutional Code (TCC) that provides robust performance within $0.5 \mathrm{~dB}$ of Shannon's limit. Windowed OFDM provides more than $30 \mathrm{~dB}$ spectrum notching. OFDM symbols with 917 usable carriers (tones) are used in conjunction with a flexible guard interval. Modulation densities from BPSK to 1024 QAM are adaptively applied to each carrier based on the channel characteristic between the transmitter $(\mathrm{Tx})$ and the receiver $(\mathrm{Rx})$.

On the MAC layer, HomePlug AV provides a Quality of Service (QoS) connection oriented, contention-free service on a periodic Time Division Multiple Access (TDMA) allocation, and a connectionless, prioritized contention-based service on a Carrier Sense Multiple Access/Collision Avoidance (CSMA/CA) allocation. The MAC receives MAC Service Data Units (MSDUs) and encapsulates them with a header, optional Arrival Time Stamp (ATS), and a checksum to create a stream of MAC frames. The stream is then divided into 512octet segments, encrypted and encapsulated into serialized PHY Blocks (PBs), and packed as MAC Protocol Data Units (MPDUs) to the PHY unit which then generates the final PHY Protocol Data Unit (PPDU) to be transmitted onto the power line [2].

HomePlug AV2 enhances significantly the capability above to accommodate new generation of multimedia applications with Gigabit performance.

2.2. The HomePlug AV2 Technology. The HomePlug system is specified for in-home communication adapted to the power line channel. The in-home communications are established with Time Division Duplexing (TDD) mechanism to allow for symmetric communication between peers, as opposed to the classical access system in ADSL with two different downstream and upstream throughputs.

The PHY layer employs OFDM modulation scheme for better efficiency and adaptability to the channel impairments (such as being frequency selective and suffering from narrowband interference and impulsive noise). The HomePlug AV2 OFDM parameters correspond to a system with 4096 carriers 


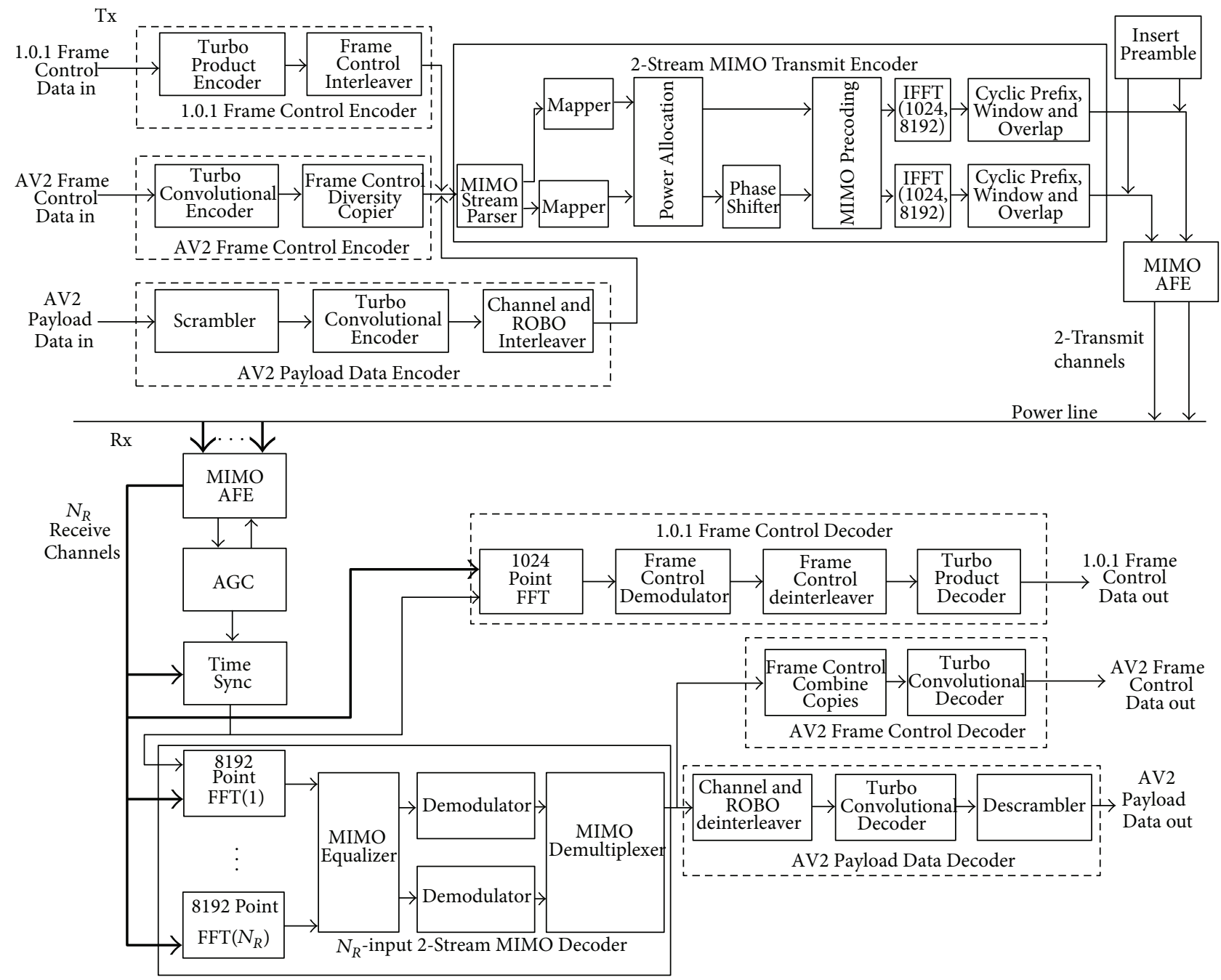

Figure 1: HomePlug AV2 transmitter and receiver PHY layer.

in $100 \mathrm{MHz}$, but only carriers from 1.8 to $86.13 \mathrm{MHz}$ are supported for communication (3455 carriers). The subcarrier spacing of $24.414 \mathrm{kHz}$ was chosen in the HomePlug AV system according to the power line coherence bandwidth characteristic and is maintained in HomePlug AV2 for interoperability.

More significantly, HomePlug AV2 incorporates MIMO capability (see details in the next sections) to improve throughput and coverage.

A block diagram of the PHY layer is shown in Figure 1. The HomePlug AV2 system is capable of supporting two network operating modes: AV-only mode and Hybrid mode (1.0.1 Frame Control Encoder in Figure 1). Hybrid mode is used for coexistence with HomePlug 1.0.1 stations. For that purpose, a 1.0.1 Frame Control Encoder is included for Hybrid modes and an AV2 Frame Control Encoder for both Hybrid and AV-only modes. The AV-only mode is used for communications in networks where only HomePlug AV and HomePlug AV2 stations are involved.
Besides, two OFDM paths are shown in order to implement the MIMO capabilities with 2 transmission ports.

Apart from the Hybrid or AV-only Frame Control symbols, the payload data can be sent using adaptive bit loading per carrier or robust modes (ROBO) with fixed Quadrature Phase Shift Keying (QPSK) constellation and several copies of data interleaved in both time and frequency.

Looking at the data path details in the block diagram (Figure 1), the following can be seen: at the transmitter side, the PHY layer receives its inputs from the MAC layer. Three separate processing chains are shown because of the different encoding for HomePlug 1.0.1 Frame Control (FC) data, HomePlug AV2 FC data, and HomePlug AV2 payload data. AV2 Frame Control data is processed by the AV2 Frame Control Encoder, which has a Turbo Convolutional Encoder and Frame Control Diversity Copier, while the HomePlug AV2 payload data stream passes through a Scrambler, a Turbo Convolutional Encoder, and an Interleaver. The HomePlug 
1.0.1 Frame Control data passes through a separate HomePlug 1.0.1 Frame Control Encoder.

The outputs of the FC Encoders and Payload Encoder lead into a common MIMO OFDM modulation structure, consisting of a MIMO Stream Parser that provides up to two independent data streams to two transmit paths which include two Mappers, a phase shifter that applies a 90degree phase shift to one of the two streams (to reduce the coherent addition of the two signals), a MIMO precoder to apply transmitter Beamforming operations, two Inverse Fast Fourier Transform (IFFT) processors, preamble and Cyclic Prefix insertion, and symbol Window and Overlap blocks, which eventually feed the AFE module with one or two transmit ports that couple the signal to the power line medium.

At the receiver, an AFE with one, two, three, or four $\left(N_{R}\right)$ receive ports operates with individual Automatic Gain Control (AGC) modules and one or more time-synchronization modules to feed separate Frame Control and payload data recovery circuits. Receivers plugged to power outlets which are connected to the three wires Line, Neutral, and Protective Earth might utilize up to three differential mode Rx ports and one common mode Rx port.

The Frame Control data is recovered by processing the received signals through a 1024-point FFT (for HomePlug 1.0.1 delimiters) and multiple 8192-point FFTs, and through separate Frame Control Decoders for the HomePlug AV2/AV and HomePlug 1.0.1 modes. The payload portion of the sampled time domain waveform, which contains only HomePlug AV2 formatted symbols, is processed through the multiple 8192-point FFT (one for each receive port), a MIMO Equalizer that receives $N_{R}$ signals, performs receive Beamforming, and recovers the two transmit streams, two Demodulators, a Demultiplexer to combine the two MIMO streams, and a channel deinterleaver followed by a Turbo Convolutional Decoder and a Descrambler to recover the AV2 payload data.

\section{PHY Layer Improvements of HomePlug AV2}

3.1. Multiple-Input Multiple-Output (MIMO) Capabilities with Beamforming. The HomePlug AV2 specification incorporates Multiple-Input Multiple-Output (MIMO) capabilities with Beamforming, which offers the benefit of improved coverage throughout the home, particularly for hard to reach outlets. MIMO technology enables HomePlug AV2 devices to transmit and receive on any two-wire pairs within a threewire configuration. Figure 2 shows a three-wire configuration with Line (L), Neutral (N), and Protective Earth (PE). Whereas HomePlug AV always transmits and receives on the Line-Neutral pair (port 1 in Figure 2), HomePlug AV2 modems can transmit and receive signals on the other pairs as well. Any two pairs formed by the Line, Neutral, or Protective Earth wires (i.e., L-N, L-PE, and N-PE) can be used at the transmitter. At the receiver, up to four receive ports may be used according to Figure 2. The common mode (CM) signal-indicated as port 4 in Figure 2 -is the voltage difference between the sum of the three wires and the ground.

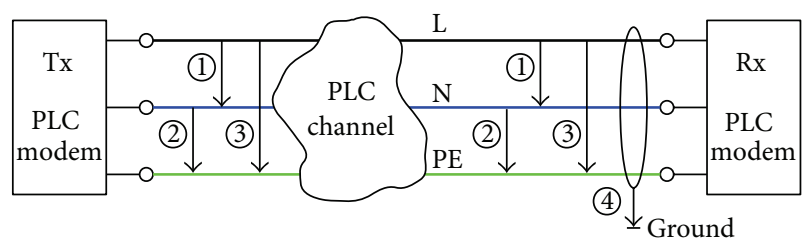

FIGURE 2: MIMO-PLC channel: different feeding and receiving options.

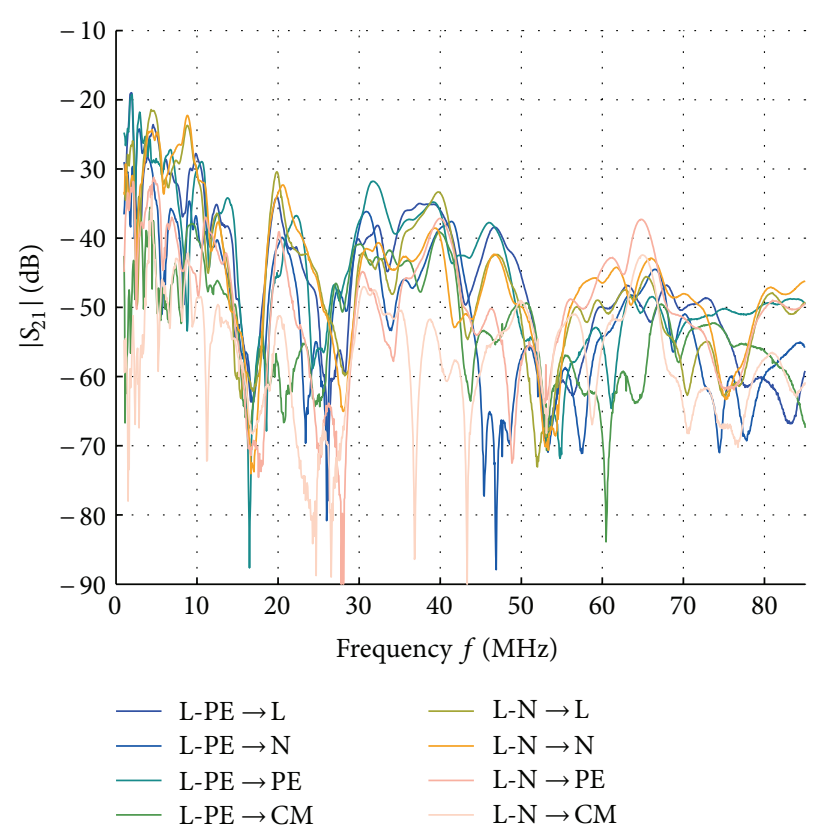

FIgURE 3: Magnitude of the transfer functions $\left(S_{21}\right.$, forward scattering parameter) of all MIMO paths of a $2 \times 4$ MIMO configuration.

The numbers of used transmit ports $N_{T}$ and used receive ports $N_{R}$ define the MIMO configuration, which is called $N_{T} \times N_{R}$ MIMO. For example, using L-N and L-PE to feed and receive signals results in a $2 \times 2$ MIMO configuration. HomePlug AV2 specification supports MIMO configurations with up to 2 Tx ports and up to $N_{R}$ Rx ports.

Some regions and maybe homes with older electrical installations do not have the third wire installed in the private buildings. In this scenario, HomePlug AV2 automatically switches to Single-Input Single-Output (SISO) operating mode. HomePlug AV2 incorporates also selection diversity in SISO mode. The ports used for feeding and receiving might be different from the traditional L-N feeding. If, for example, the path from L-PE to L-N offers better channel characteristics than L-N to L-N, the transmitter can choose to use the L-PE port for feeding.

Figure 3 shows the magnitude of several transfer functions of a typical MIMO-PLC channel. The figure illustrates all eight possible paths of a $2 \times 4$ MIMO configuration where $\mathrm{L}-\mathrm{N}$ and L-PE pairs are used as feeding and all four receive ports are used as receiving. The example shows that the signal 


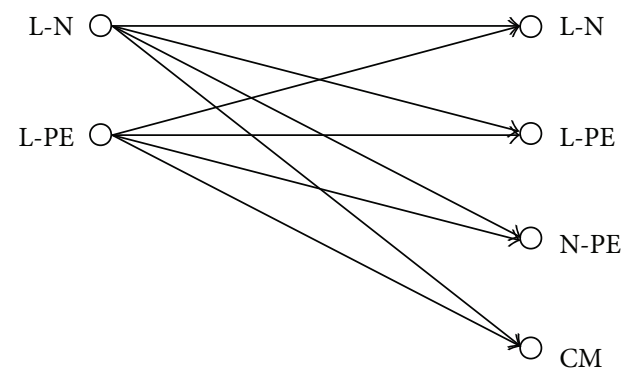

(a)

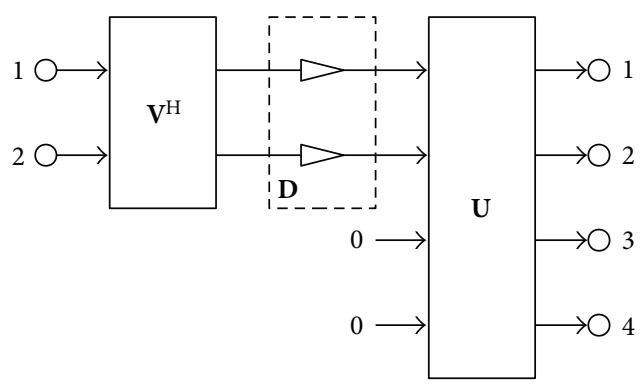

(b)

FIGURE 4: Schematic MIMO channel and decomposition into parallel and independent MIMO streams.

fed into one port is visible at all receive ports. Crosstalk caused by the coupling of the wires results in the presence of all possible MIMO paths. At most frequencies the $S_{21}$ (forward scattering parameter of the network analyzer) curves are quite independent. As less correlation the $S_{21}$ curves show, as higher is the gain by MIMO. For some frequencies, for example in the region of the fading degradation around $16 \mathrm{MHz}$, the shapes of the transfer functions are similar due to the same underlying topology of the different MIMO paths. Usually, the wires are located in parallel within the walls facing a similar multipath propagation. This results in a higher spatial correlation of the MIMO-PLC channel than compared to radio MIMO channels.

More information about the MIMO-PLC channel characteristics and channel modeling may be found in [6-18].

The channels presented in Figure 3 were recorded with a delta coupler at the transmitter (L-PE, L-N) and a star coupler at the receiver $(\mathrm{L}, \mathrm{N}, \mathrm{PE}, \mathrm{CM})$. This coupler combination allows $2 \times 4$ MIMO [19].

The MIMO-PLC channel is described by a $N_{R} \times N_{T}$ channel matrix for each OFDM subcarrier $c$ :

$$
\mathbf{H}(c)=\left[\begin{array}{ccc}
h_{11}(c) & \cdots & h_{1 N_{T}}(c) \\
\vdots & \ddots & \vdots \\
h_{N_{R} 1}(c) & \cdots & h_{N_{R} N_{T}}(c)
\end{array}\right]
$$

with $c$ belonging to the set of used subcarriers (depending, e.g., on the frequency band and tone mask).

Figure 4(a) shows the schematic MIMO channel for a $2 \times 4$ MIMO configuration with the overall 8 MIMO paths. HomePlug AV2 supports 1 or 2 streams (see Section 3.1.1). As HomePlug AV2 supports MIMO configurations with up to 2 Tx ports and up to $N_{R}$ Rx ports, the maximum number of supported streams in AV2 is 2. The number of the underlying MIMO streams depends on the rank of the channel matrix. If the channel matrix has full rank, the number of MIMO streams is $=\min \left(N_{T}, N_{R}\right)$. The underlying MIMO streams are obtained by a Singular Value Decomposition (SVD) of the channel matrix [20]:

$$
\mathbf{H}(c)=\mathbf{U}(c) \mathbf{D}(c) \mathbf{V}(c)^{H},
$$

where $\mathbf{V}$ and $\mathbf{U}$ are unitary matrices, that is, $\mathbf{V}^{-1}=\mathbf{V}^{H}$ and $\mathbf{U}^{-1}=\mathbf{U}^{H}$ (with $H$ the Hermitian operator) and $\mathbf{D}$

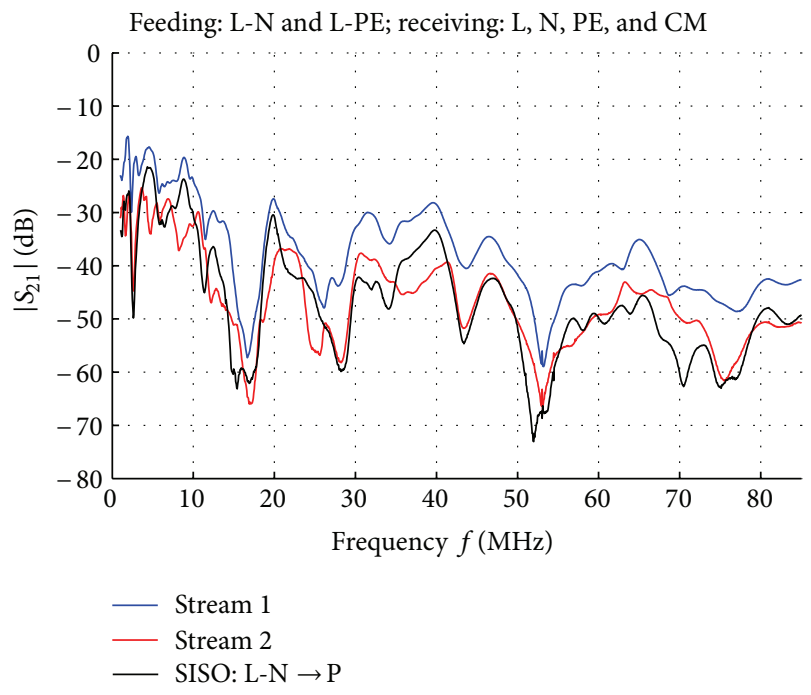

Figure 5: Attenuation of the decomposed PLC-MIMO channel of Figure 3 .

is a diagonal matrix containing the singular values of $\mathbf{H}$. Figure 4(b) illustrates this decomposition.

Figure 5 shows the decomposition of the two streams of the MIMO-PLC channel shown in Figure 3. As a comparison, the attenuation of the SISO channel is also depicted.

The decomposition into parallel and independent streams by means of the SVD illustrates the MIMO gain; instead of having one spatial stream in SISO, two independent spatial streams are available in a $2 \times N_{R}$ MIMO configuration with $N_{R} \geq 2$ doubling in average the MIMO capacity $[9,12,13,21]$. This fact is also verified by means of Figure 5; the first spatial stream shows less attenuation compared to the SISO channel; the second stream is slightly higher attenuated compared to SISO. Hence, in this example, the MIMO link would result in more than twice the capacity of the SISO link.

3.1.1. MIMO Stream Parser. Depending on how many streams are used in the transmission, the payload bits have to be split into different spatial streams. This task is performed by the MIMO Stream Parser (MSP). The MSP splits the incoming 
bits into one or two streams based on the MIMO mode of operation and the Tone Map information (see Figure 1). To transmit a single-stream payload with Spot-Beamforming (see Section 3.1.3) or for SISO transmissions, the MSP sends all the data at its input to the first Mapper. In this case, the MSP operates as if it had only one output and it was connected to the first mapper in Figure 1. To transmit a twostream payload with Eigen-Beamforming (see Section 3.1.3), the MSP allocates the bits to the two streams.

3.1.2. Precoding. Precoded Spatial Multiplexing or Beamforming was chosen as the MIMO scheme as it offers the best performance by adapting the transmission in an optimum way to the underlying Eigen modes of the MIMOPLC channel. The best performance is achieved for various channel conditions. On the one hand, the full spatial diversity gain is achieved in highly attenuated and correlated channels when each symbol is transmitted via each available MIMO path. On the other hand, a maximum bit rate gain is achieved for channels with low attenuation when all available spatial streams are utilized. Beamforming also offers flexibility with respect to the receiver configuration. Only one spatial stream may be activated by the transmitter when only one receive port is available, that is, if the outlet is not equipped with the 3rd wire or if a simplified receiver implementation is used that supports only one spatial stream. Since Beamforming aims to maximize one MIMO stream, the performance loss of not utilizing the second stream is relatively small compared to the Spatial Multiplexing schemes without precoding. This is especially true for highly attenuated and correlated channels, where the second MIMO stream carries only a small amount of information. These channels are most critical for PLC and adequate MIMO schemes are important. A comparison and analysis of different MIMO schemes may be found in $[14,21,22]$.

Beamforming requires knowledge about the channel state information at the transmitter to apply the optimum precoding. Usually, only the receiver has channel state information, for example, by channel estimation. Thus, the information about the precoding has to be fed back from the receiver to the transmitter. The HomePlug AV2 specification supports adaptive modulation [23, 24]. The application of adaptive modulation also requires feedback about the constellation of each subcarrier (Tone Map); that is, the feedback path is required anyway. The information about the Tone Maps and the precoding are updated simultaneously upon a change in the PLC channel (realized by a channel estimation indication message). The information about the precoding is quantized very efficiently and the amount required for the precoding information is in the same order of magnitude as the information about the Tone Maps. Thus, the overhead in terms of management messages and required memory can be kept low.

The optimum linear precoding matrix $\mathbf{F}$ for a precoded Spatial Multiplexing system can be factored into the two matrices $\mathbf{V}$ and $\mathbf{P}$ [25]:

$$
\mathbf{F}=\mathbf{V P}
$$

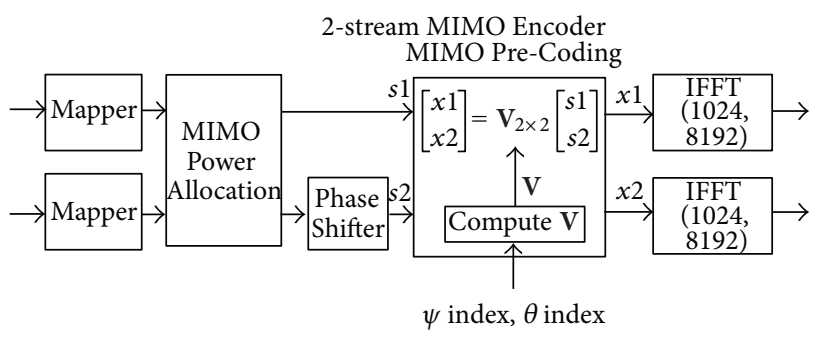

FIgURE 6: Precoding at the transmitter.

Note that the subcarrier index is omitted in (3) and in the following section to simplify the notation and to allow better legibility. However, it has to be kept in mind that the following vector and matrix operations are applied to each subcarrier separately if not stated otherwise.

$\mathbf{P}$ is a diagonal matrix that describes the power allocation of the total transmit power to each of the transmit streams. Power allocation is considered in more detail in Section 3.1.4. $\mathrm{V}$ is the right hand unitary matrix of the SVD of the channel matrix (see (2)). Precoding by the unitary matrix $\mathbf{V}$ is often referred to as unitary precoding or Eigen-Beamforming.

Figure 6 shows the basic MIMO blocks of the transmitter. The Mappers of the two streams follow the MIMO stream parser (see Figure 1). The two symbols of the two streams are then weighted by the power allocation coefficients and multiplied by the matrix $\mathbf{V}$ (the computation of $\mathbf{V}$ is explained in Section 3.1.3 in more detail). Finally, each stream is OFDM modulated separately.

3.1.3. Beamforming and Quantization of the Beamforming Matrix. The precoding matrix $\mathbf{V}$ is derived by means of the SVD (see (2)) from the channel matrix $\mathbf{H}$.

There are two possible modes of operation. If only one spatial stream is utilized, single-stream Beamforming (or Spot-Beamforming) is applied. In this case, the precoding is described by the first column vector of $\mathbf{V}$; that is, the precoding simplifies to a column vector multiplication. Note that despite the fact that only one spatial stream is used, both transmit ports are active as the precoding vector splits the signal to two transmit ports. If both spatial streams are used, two-stream Beamforming (or Eigen-Beamforming) is used and the full precoding matrix $\mathbf{V}$ is applied in the MIMO precoding block in Figure 6.

Since the information about the precoding matrix has to be fed back from the receiver to the transmitter, an adequate quantization is required. To achieve this goal, the special properties of $\mathbf{V}$ are utilized.

The unitary property of $\mathbf{V}$ consequences that the columns $\mathbf{v}_{i}(i=1,2)$ of $\mathbf{V}$ are orthonormal; that is, the column vectors are orthogonal and the norm of each column vector is equal to 1 [26]. There is more than one unique solution to the SVD; the column vectors of $\mathbf{V}$ are phase invariant; that is, multiplying each column vector of $\mathbf{V}$ by an arbitrary phase rotation results in another valid precoding matrix. 

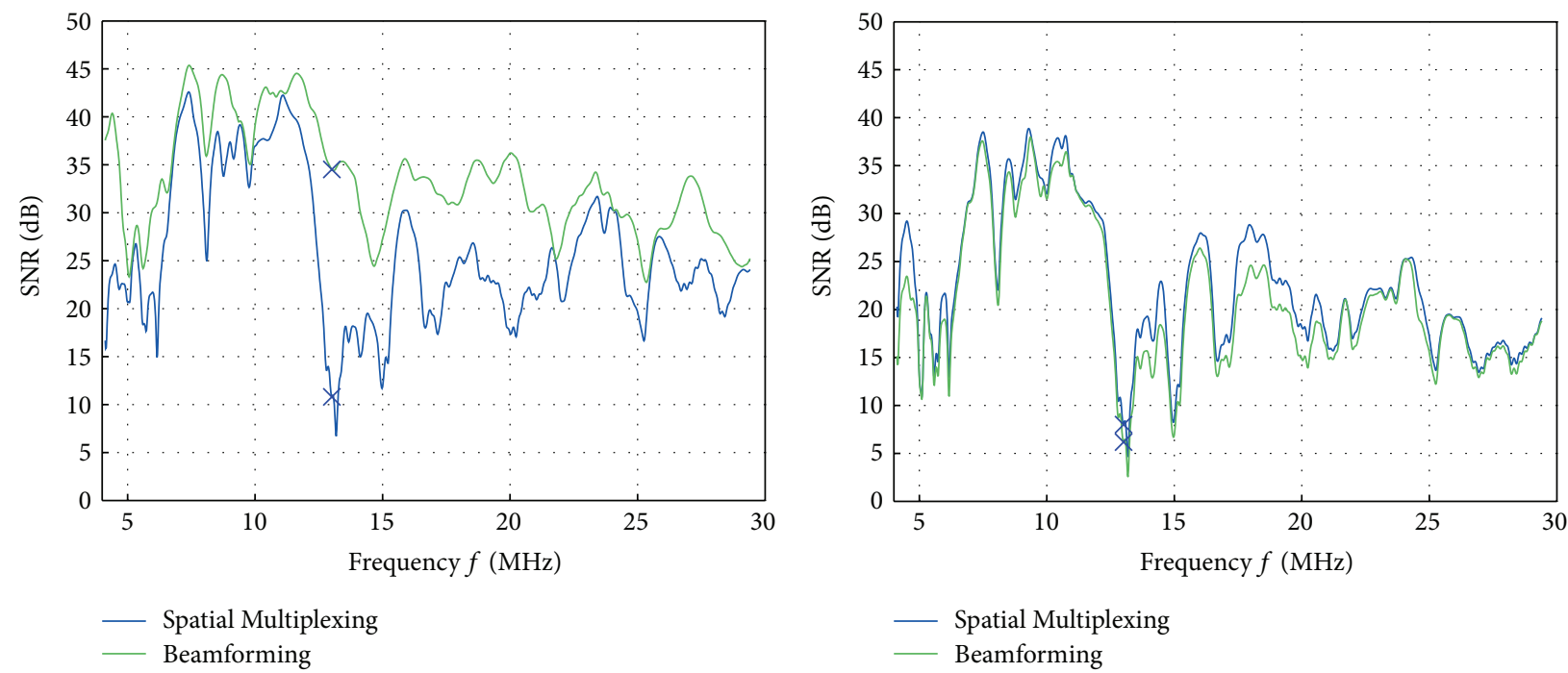

(a)

(b)

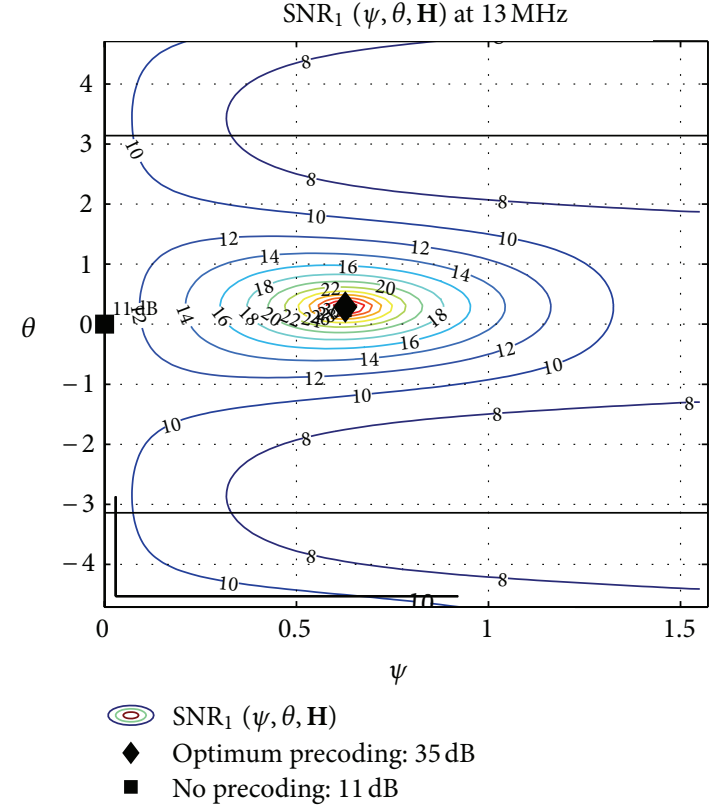

(c)

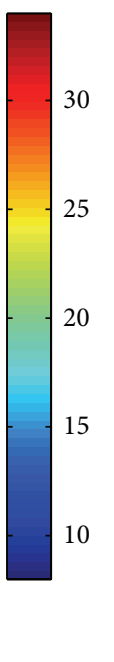

25

20

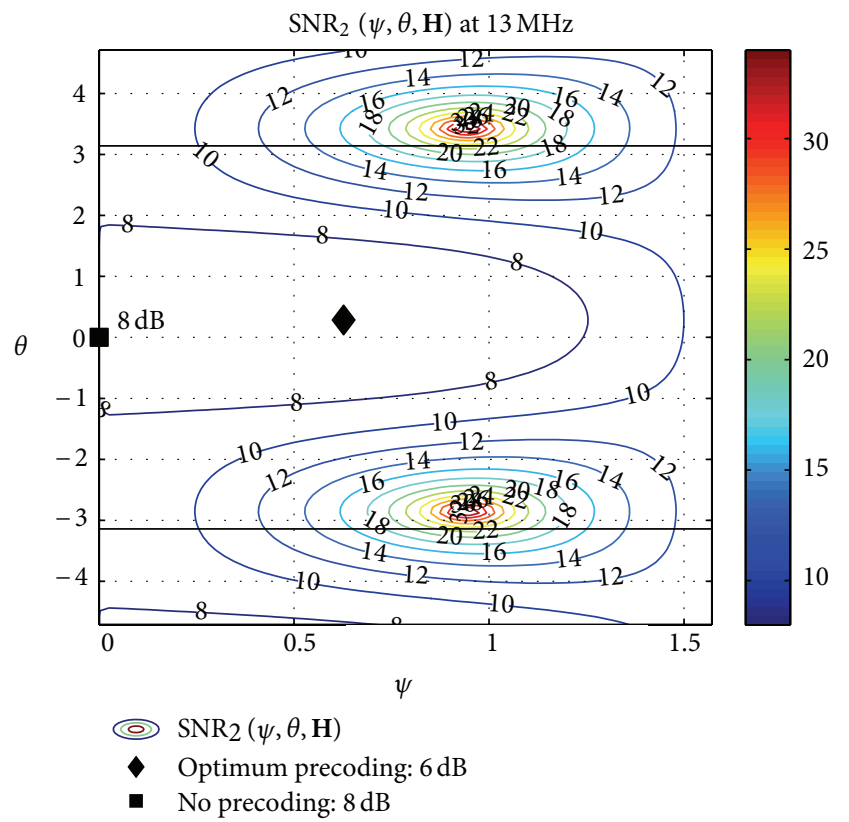

(d)

FIGURE 7: Influence of precoding on the SNR: SNR of the first (a) and second (b) streams with and without precoding, transmit power to noise power of $\rho=65 \mathrm{~dB}, 40 \mathrm{~dB}$ average channel attenuation; SNR depending on the precoding matrix at $13 \mathrm{MHz}$ of the first (c) and second (d) streams.

These properties allow to represent the complex $2 \times 2$ matrix $\mathbf{V}$ by only the two angles $\theta$ and $\psi$ :

$$
\mathbf{V}=\left[\begin{array}{ll}
\mathbf{v}_{1} & \mathbf{v}_{2}
\end{array}\right]=\left[\begin{array}{ll}
v_{11} & v_{12} \\
v_{21} & v_{22}
\end{array}\right]=\left[\begin{array}{cc}
\cos \psi & \sin \psi \\
-e^{j \theta} \sin \psi & e^{j \theta} \cos \psi
\end{array}\right],
$$

where the range of $\theta$ and $\psi$ to represent all possible Beamforming matrices is $0 \leq \psi \leq \pi / 2$ and $-\pi \leq \theta \leq \pi$.

According to the phase-invariance property, the first entry of each column $\left(v_{11}, v_{12}\right)$ may be set to be real without loss of generality, as defined in (4). It is easy to prove that the properties of the unitary precoding matrix are fulfilled.
The norm of the column vectors is one: $\left|v_{11}\right|^{2}+\left|v_{21}\right|^{2}=$ $\left|v_{12}\right|^{2}+\left|v_{22}\right|^{2}=\sin ^{2}(\psi)+\cos ^{2}(\psi)=1$. Also, the two columns are orthogonal:

$$
\mathbf{v}_{1}^{H} \mathbf{v}_{2}=\sin (\psi) \cos (\psi)-\sin (\psi) \cos (\psi) e^{-j \theta} e^{j \theta}=0 .
$$

In both modes, Spot-Beamforming and EigenBeamforming, the Beamforming vector or Beamforming matrix, respectively, is described by both angles $\theta$ and $\psi$. Thus, the signaling of $\theta$ and $\psi$ is the same in both modes. 
If the MIMO Equalizer is based on zero-forcing (ZF) detection, the detection matrix

$$
\mathbf{W}=\mathbf{H}^{p}=\left(\mathbf{H}^{H} \mathbf{H}\right)^{-1} \mathbf{H}^{H}
$$

is the pseudo inverse $\mathbf{H}^{\mathrm{P}}$ of the channel matrix $\mathbf{H}$. In case of Eigen-Beamforming with the precoding matrix $\mathbf{V}, \mathbf{H}$ can be replaced by the equivalent channel $\mathrm{HV}$ in (6) and the detection matrix can be expressed by

$$
\mathbf{W}=\mathbf{V}^{H} \mathbf{H}^{p}=\mathbf{D}^{-1} \mathbf{U}^{H} .
$$

The SNR of the MIMO streams after detection is calculated as

$$
\mathrm{SNR}_{1}=\rho \frac{1}{\left\|\mathbf{w}_{1}\right\|^{2}}, \quad \mathrm{SNR}_{2}=\rho \frac{1}{\left\|\mathbf{w}_{2}\right\|^{2}},
$$

with $\rho$ being the ratio of transmit power to noise power and $\left\|w_{i}\right\|$ the norm of the $i$ th row of the detection matrix $\mathbf{W}$.

Figure 7(a) shows the SNR of the first stream of a MIMOPLC channel. The median attenuation of this link is $40 \mathrm{~dB}$. The ratio of the transmit power to noise power is $\rho=65 \mathrm{~dB}$. The blue line represents the SNR of Spatial Multiplexing without precoding and the green line represents the EigenBeamforming. The SNR of the 2nd stream is displayed in Figure 7(b).

The two markers $\mathrm{X}$ in Figure 7(a) mark a frequency $(13 \mathrm{MHz})$ where good Beamforming conditions are found. Different precoding matrices influence the SNR of the two MIMO streams according to (6) and (8).

Figures $7(\mathrm{c})$ and $7(\mathrm{~d})$ show the level of the gain or signal elimination due to Beamforming for the frequency marked by $\mathrm{X}$ in Figures 7(a) and 7(b). The color lines in Figures 7(c) and $7(\mathrm{~d})$ indicate the SNR. Depending on the precoding matrix the SNR varies between $6 \mathrm{~dB}$ and $35 \mathrm{~dB}$. No Beamforming $(\psi=0$ and $\theta=0)$ would result in a SNR of $11 \mathrm{~dB}$. As seen in Figure 7(c), there is one SNR maximum in the area spanned by $\psi$ and $\theta$. Due to both streams being orthogonal one shows an SNR minimum at the location where the other one has its maximum. The SNR plot in Figures 7(c) and 7(d) is $2 \pi$ periodic in $\theta$, where the black horizontal lines indicate $\theta= \pm \pi$.

The Beamforming matrix is quantized efficiently to reduce the amount of feedback required to signal $\mathrm{V}$. This quantization is chosen such that the loss of the SNR after detection (refer also to (6) and (8)) is within $0.2 \mathrm{~dB}$ compared to optimum Beamforming without quantization.

3.1.4. Power Allocation. MIMO power allocation is applied to two-stream MIMO transmissions. The power allocation adjusts the power of a carrier on one stream relative to the other stream. For SISO transmissions and MIMO SpotBeamforming transmissions, MIMO power allocation can be bypassed since there is only one transmit stream. In this case, the only available option is to allocate all the power to the single stream. The power allocation module is located between the Mapper and the precoding block (see Figure 1) and performs on the two MIMO streams before EigenBeamforming. The power allocation in AV2 is designed in a way not to feed back additional information from the receiver to the transmitter. The power allocation evaluates the Tone Maps of the two streams to set the power allocation coefficients of the two streams. It was shown that the performance is very close to the optimum power allocation (mercury water filling [27]). Power allocation improves especially highly attenuated channels at the high coverage point.

3.1.5. Precoding Grouping. HomePlug AV2 devices support an expanded frequency spectrum (up to $86.13 \mathrm{MHz}$, see Section 3.2) and one additional stream (utilizing MIMO) compared to the $30 \mathrm{MHz}$ for HomePlug AV devices. Thus, HomePlug AV2 devices need to store two Tone Maps (one for each stream) and one precoding matrix (PCM) per carrier. This significantly increases the memory requirements for an AV2 modem. In order to save memory, HomePlug AV2 devices transmit and store the PCM only on a subset of carriers called precoding pilot carriers. At the transmitter, the PCMs for the carriers between two adjacent precoding pilot carriers are obtained via interpolation. The spacing between the precoding pilot carriers is selected out of a set of predefined values and may be adjusted depending on the memory capabilities or channel conditions. The more memory is embedded in the modems, the finer the granularity is. The performance loss of precoding grouping compared to the quantization of each subcarrier separately is marginal due to the high correlation of the precoding matrices of neighbored subcarriers. An investigation about different precoding grouping algorithms for MIMO-PLC may be found in [14].

3.2. Extended Frequency Band up to $86 \mathrm{MHz}$. During the specification development, the AV TWG realized a measurement campaign, where power line channel and noise measurements were performed in 30 homes located in different countries, from Europe to USA. Such variability was a key ingredient to obtain insight into the power line at frequencies above those used in HomePlug AV (1.8-30 MHz). In particular, in every home, all the possible links among at least 5 different nodes were measured.

Examples of recorded channel attenuation and noise PSD are reported in Figures 8 and 9, respectively.

Based upon the results of the measurement campaign, the present EMC regulations, coverage, and complexity targets, the final outcome of the analysis was the selection of the 30$86 \mathrm{MHz}$ band for the following reasons.

(i) The FM band region $87.5-108 \mathrm{MHz}$ shall be avoided. In fact this frequency region presents higher attenuation and higher noise compared to the $30-86 \mathrm{MHz}$ band (see also Figures 8 and 9). Since, probably, lower transmit level requirements will be needed in order to not interfere with the FM radio service, very low operating SNRs are obtained in this band with negligible coverage increase;

(ii) The $30-86 \mathrm{MHz}$ frequency band appears to offer a throughput increase especially at the low to mid coverage percentages; the reason is that while the 


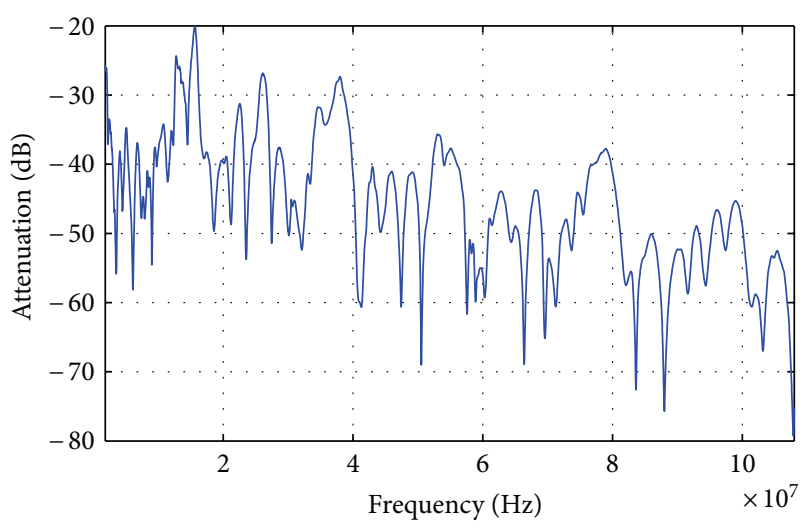

Figure 8: HomePlug AV2 measurement campaign. Example of channel measurement.

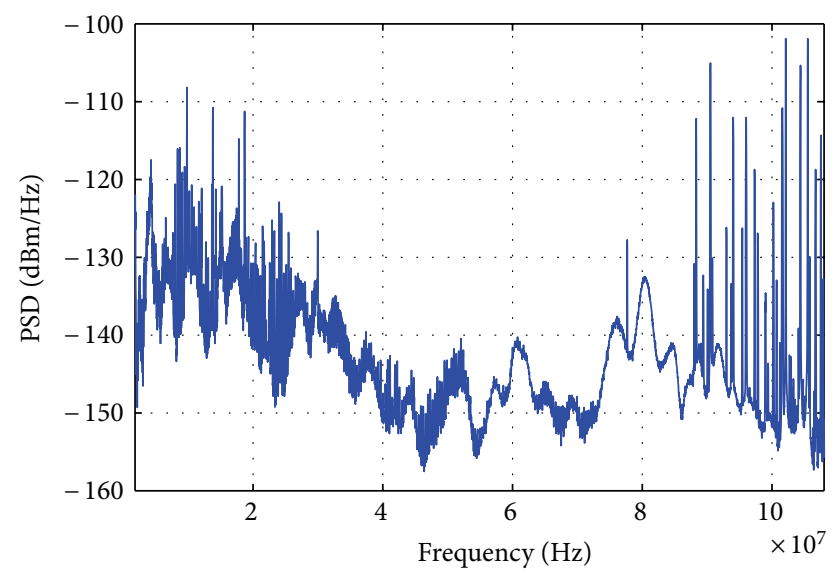

Figure 9: HomePlug AV2 measurement campaign. Example of noise measurement.

attenuation is greater compared to the $1.8-30 \mathrm{MHz}$ band, noise is lower. A concern derives from the fact that EMC requirements for the $30-86 \mathrm{MHz}$ frequency band are generally tighter than in the $1.8-30 \mathrm{MHz}$ band. However, this observation could be mitigated in view of the fact that, for instance, the upcoming standard FprEN 50561-1 appears to be more restrictive in the $1.8-30 \mathrm{MHz}$ frequency band.

(iii) Within the HomePlug AV2 specification it has been possible to provide flexibility in choosing the stop frequency in the $30-86 \mathrm{MHz}$ interval. In particular, an AV2 device implementing a frequency band 1.8$X \mathrm{MHz}$ band (with $30<X<86$ ) will be interoperable with a device implementing a frequency band 1.8- $Y \mathrm{MHz}$ (with $30<Y<86, X \neq Y$ ).

(iv) The $30-86 \mathrm{MHz}$ frequency band extension allows devices to be fully interoperable with the IEEE 1901 devices that use the $1.8-50 \mathrm{MHz}$ frequency band. In fact, HomePlug AV2 devices that implement a frequency band extension shall support at least the IEEE 1901 bandwidth.

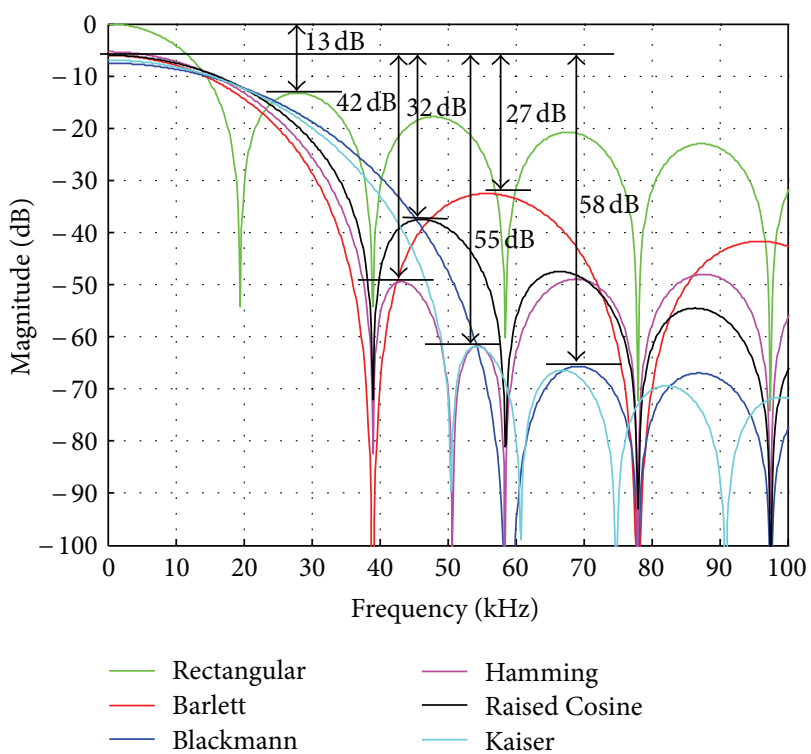

FIgURE 10: OFDM side lobes of a single carrier. Comparison of various window functions.

3.3. Efficient Notching. HomePlug AV2 increases throughput by allowing devices to minimize the overhead incurred due to EMC notching requirements. While in HomePlug AV the mechanism ("windowed OFDM") for creating the PSD notches is fixed and relatively conservative, HomePlug AV2 devices may gain up to $20 \%$ in efficiency if they implement additional techniques to accommodate sharper PSD notches. The $20 \%$ includes the gain of guard carriers which were excluded by HomePlug AV modems and the reduced Transition Interval in time domain. Such devices gain additional carriers at the band edges and may utilize shorter cyclic extensions, which reduces the duration of the OFDM symbols.

3.3.1. Influence of Windowing on Spectrum and Notch Shape. The FFT process uses a rectangular window to cut data out of a continuous stream to convert them from time to frequency domain. The FFT of a rectangular function in the time domain is a $\sin (x) / x$ function in the frequency domain. The $\sin (x) / x$ becomes 0 at integer multiples of $\pi$. Some parts of the signal remain in-between the zeros, which results in the unwanted side lobes of an FFT OFDM system. Figure 10 shows a $\sin (x) / x$ function as green curve. The frequency axis is shown horizontally. The level in a logarithmic view is presented on the vertical axis.

The process of multiplying a window with an OFDM symbol (see Figure 11) in the time domain aims to suppress the sharp corners at the beginning and the end of the OFDM symbol in order to get smooth transitions. This affects the shape and distances of the side lobes in the frequency domain [28]. There are numerous types of window functions available for implementation such as Hamming, Barlett (triangular), Kaiser, Blackman, Raised Cosine (Hann), and so forth. A comparison of various window waveforms with the achieved side lobe attenuation is shown in Figure 10 which also serves 


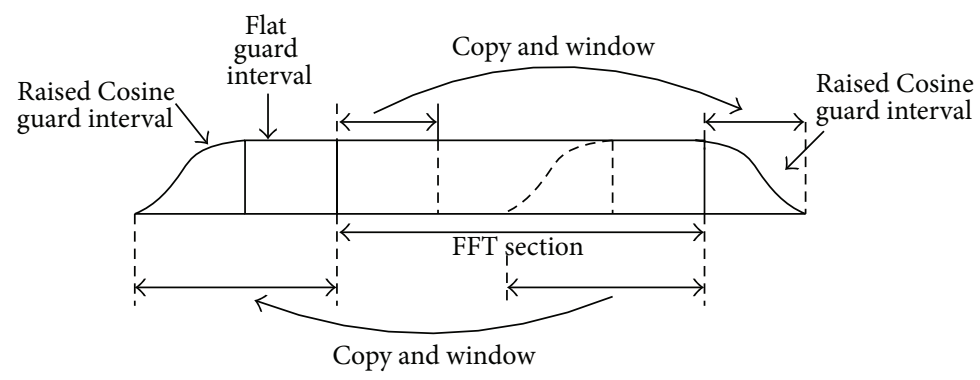

FIGURE 11: OFDM symbol with GI and window.

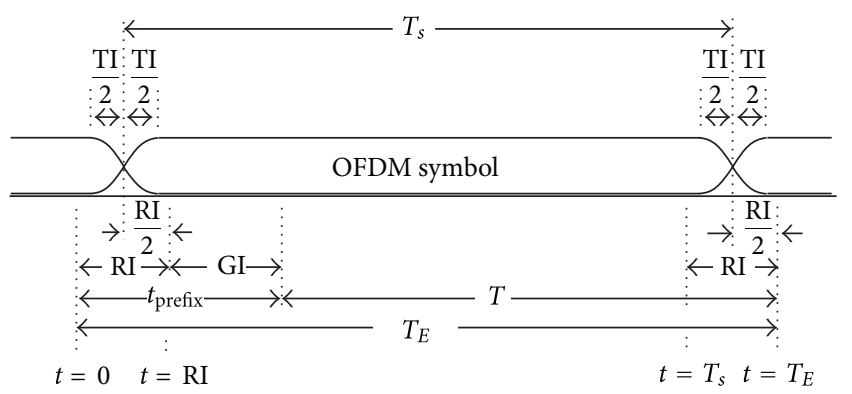

FIGURE 12: Consecutive OFDM symbols, guard interval (GI), roll-off interval (RI), and windowing.

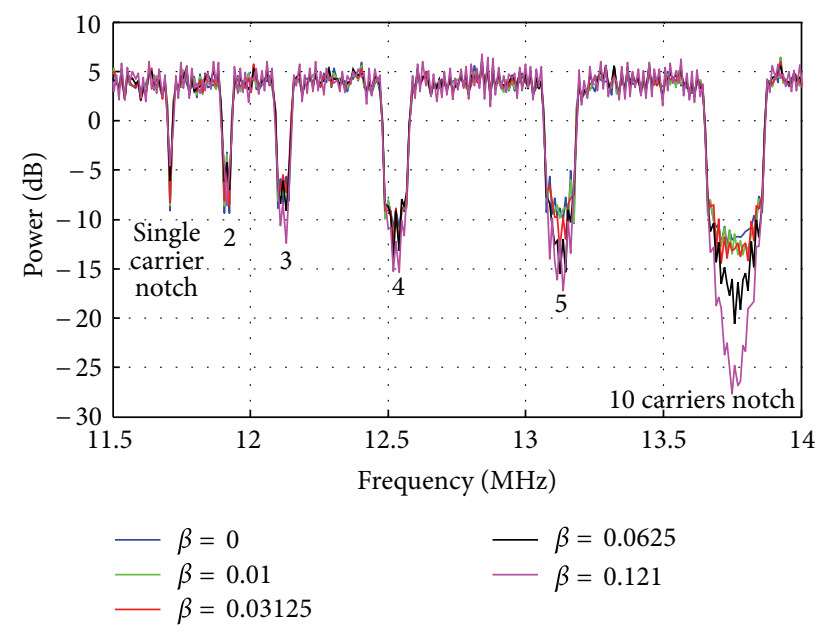

FIGURE 13: OFDM spectra with different notch widths and depths achieved with different roll-off factors.

to illustrate the disadvantage of windowing. The side lobes are suppressed, but the main lobe stays wider. The first time the spectrum reaches zero, the distance for all windows is at least twice the frequency of the rectangular window used by the pure FFT. Windowing is considered to be state of the art in signal processing [29].

The process of how a window is applied is shown in Figure 11. The original OFDM symbol or the output of the IFFT at the transmitter is marked with "FFT section." As described above, the guard interval is copied from the tail samples to the beginning of the symbol. In order to create a window, the symbol has to be expanded further at the beginning and at the end by copying the bits as done for the Guard Interval (GI). This expansion is multiplied with the smoothly descending window. The more smoothly the signal approaches zero in the time domain, the lower the side lobes in the frequency domain. Of course, this expansion of a symbol is a waste of communication resources as it does not carry useful information and has to be cut by the receiver.

The two descending slopes in the time domain could overlap, to save communication resources at consecutive OFDM symbols, as shown in Figure 12. The new symbol time $T_{s}$ is measured between the middles of the roll-offs before and after the symbol. The overlap region is the roll-off interval (RI) (see in Figure 12) which is related to the OFDM symbol duration $T_{s}$ via the parameter $\beta$ :

$$
\mathrm{RI}=\beta T_{\mathrm{s}}
$$

and the total symbol length $T_{E}$ :

$$
T_{E}=(1+\beta) \cdot T_{s} .
$$

The HomePlug AV specification allows the usage of several Guard intervals [30, 31]. If the shortest guard interval is selected, HomePlug $\mathrm{AV}$ uses a $\beta$ of $\mathrm{RI} /(T+\mathrm{GI})=$ $4.96 \mu s /(40.96 \mu s+5.56 \mu s)=0.1066$.

With HomePlug AV2, the Transition Interval TI is introduced. The shape of the windowing is transmitter implementation dependent; it does not affect interoperability. The pulse shaping window and Guard interval might be reduced even to zero to minimize overhead in time domain and to notch efficiently. In order to guarantee backward compatibility with previous HomePlug versions the definition and timings of the parameter RI as shown in Figure 12 have to stay stable. In contrast to RI, the new parameter TI might be reduced to zero.

There is a balance between this intervals and obtaining a better side lobe attenuation.

Figure 13 shows the notch of any OFDM system spectrum which could be obtained by varying the roll-off factors (the $\beta$ values) of the Raised Cosine window. Increasing the roll-off factor directly increases the amount of attenuation achieved inside the notches. However, this has the drawback of increased symbol length.

To create notches in the OFDM spectrum, a model is implemented using QAM modulation and notches of omitting a various number of carriers: $1,2,3,4,5$, and 10. A max-hold function is implemented in order to create these 
figures. The 10 carrier notch shows the spectral benefit of windowing. The influence of windowing is hardly visible up to the point of the 5-carrier notch. At the 5-carrier notch, the difference between no window and the highest simulated $\beta$ is around $5 \mathrm{~dB}$. The trade-off for this $\beta$ is a $27 \%$ longer symbol time. At the 10-carrier notch, the center frequency is suppressed by more than $15 \mathrm{~dB}$ without windowing, but the adjacent side slopes of the used corner carriers are only improved by $5 \mathrm{~dB}$.

The additional overhead in the time domain is extremely large compared to the improved notch depth. Windowing with a small roll-off factor is sufficient in order to suppress the side lobes outside the used spectrum, but it is not recommended to increase the depth of a single carrier notch.

In the case of the HomePlug AV specification using a $\beta$ of 0.1066 , some guard carriers on the left and right side of a protected frequency range have to be omitted to guarantee the depth of the notch. The North American Carrier Mask requests 10 notches in-between $1.7 \mathrm{MHz}$ and $30 \mathrm{MHz}$. The first carriers as well the last carriers of this spectrum are notched. These two notches have only one slope to the carriers allocated for communications. All other notches have two slopes resulting in 18 notch slopes in total. The spectrum loses almost $6 \%$ of communication resources in frequency domain. Additionally the $\beta$ causes almost $13 \%$ of wasted resources in time domain. Assuming an ideal implementation with maximal sharpness in time as well as frequency domain, it would be possible to regain these communication resources when applying the North American Carrier Mask. If the frequency spectrum becomes more fragmented because of additional notches like requested by the newly upcoming European regulations [32] these losses become even more obvious.

3.3.2. Digital, Adaptive Band Stop Filters Improve the Notch's Depth and Slopes. Of course an ideal implementation as described above is not possible, but digital band stop filters increase the sharpness of the notches as well the implementation efforts in hardware. Shrinking the semiconductor manufacturing process to smaller structures, allowing to integrate additional functions on the same die size, shifts the balance towards hardware implementation efforts.

HomePlug AV2 specification gives maximum freedom to the chip implementer. Filter algorithm, order, and structure are implementation dependent. An example is documented in [19]. As higher the filtering efforts, as better is the sharpness of the notch slopes, as shorter might be GI, as higher the resources available for communication. A reduction of the Guard Interval down to zero (see Section 3.5.1) is possible at short PLC channels without multipath reflections or intersymbol interference.

3.4. HomePlug AV2 Power Optimization Techniques. The HomePlug AV2 standard introduces two novel techniques that can be used to optimize the use of transmit power. The first one, named "transmit power back-off", is a technique that reduces the transmitted power spectral density for a selected set of carriers when this can be done without adversely affecting performance. Conversely, the second technique, called "EMC Friendly Power Boost" is a technique that allows the transmitter to increase the power on some carriers with the knowledge that this can be done without exceeding regulatory limits.

3.4.1. Power Back-Off. In power line communications the transmit power limit is typically defined as a power spectral density (PSD) mask applicable over the range of frequencies used in the standard. And since power line modems are directly connected to the electrical wiring, they are traditionally designed to transmit with the maximum allowed transmit PSD on each frequency (i.e., they do not need to be sensitive to a limited battery supply). In many cases maximizing transmit power leads to the best performance; however, certain definitions of PSD masks combined with certain channel conditions can produce cases where modems can benefit from transmitting at less than the maximum allowed power level.

We illustrate the benefits of transmit power control using the North American regulatory limits as an example. FCC regulations that are applicable to power line devices in North America are commonly interpreted to allow a transmit PSD of $-50 \mathrm{dBm} / \mathrm{Hz}$ from 1.8 to $30 \mathrm{MHz}$, and $-80 \mathrm{dBm} / \mathrm{Hz}$ from $30 \mathrm{MHz}$ up to $86 \mathrm{MHz}$. This $30 \mathrm{~dB}$ drop in the PSD (at $30 \mathrm{MHz}$ ) causes the signal from the higher frequency carriers (above $30 \mathrm{MHz}$ ) to have much smaller amplitude than the signal for the low band carriers (up to $30 \mathrm{MHz}$ ). Consequently, when the overall signal is represented in a quantized digital domain, the high band signal has lower resolution than the signal in the lower band and will therefore also have a limited SNR. This will be evident at the transmitter where the $30 \mathrm{~dB}$ drop will result in a reduction of 5 bits of resolution for the high band signal. If the transmit power is backed off in the low band, so that the PSD drop is reduced, then the high band signal will be represented with an increased number of bits of resolution and enjoy increased SNRs out of the transmitter.

Another limiting factor is the limited dynamic range of the analog to digital converter (ADC). We illustrate its impact using once again the example of the North American PSD limits. For the sake of simplicity, we assume a flat power line channel, and flat noise spectrum in Figure 14. For convenience, the power line channel (cyan curves labeled Rx signal: the transmitted signal after channel attenuation) and noise (green curves labeled $\mathrm{Rx}$ noise) contributions to the received signal are shown separately. Moreover, the different signals are shown before (dashed line curves) and after (continuous line curves) the analog amplifier. On the left of Figure 14 the scenario without power back-off is shown; the transmitted signal is $30 \mathrm{~dB}$ greater in the $1.8-30 \mathrm{MHz}$ band compared to the $30-86 \mathrm{MHz}$ band. The analog amplifier brings the received signal to a level A tailored to optimize the ADC conversion. Since the dominating noise is the ADC noise (black curve), after the ADC converter SNRs of $35 \mathrm{~dB}$ and $5 \mathrm{~dB}$ are found below and above $30 \mathrm{MHz}$, respectively. On the right of Figure 14 the scenario with power backoff is shown: the transmitted signal is reduced by $10 \mathrm{~dB}$ 


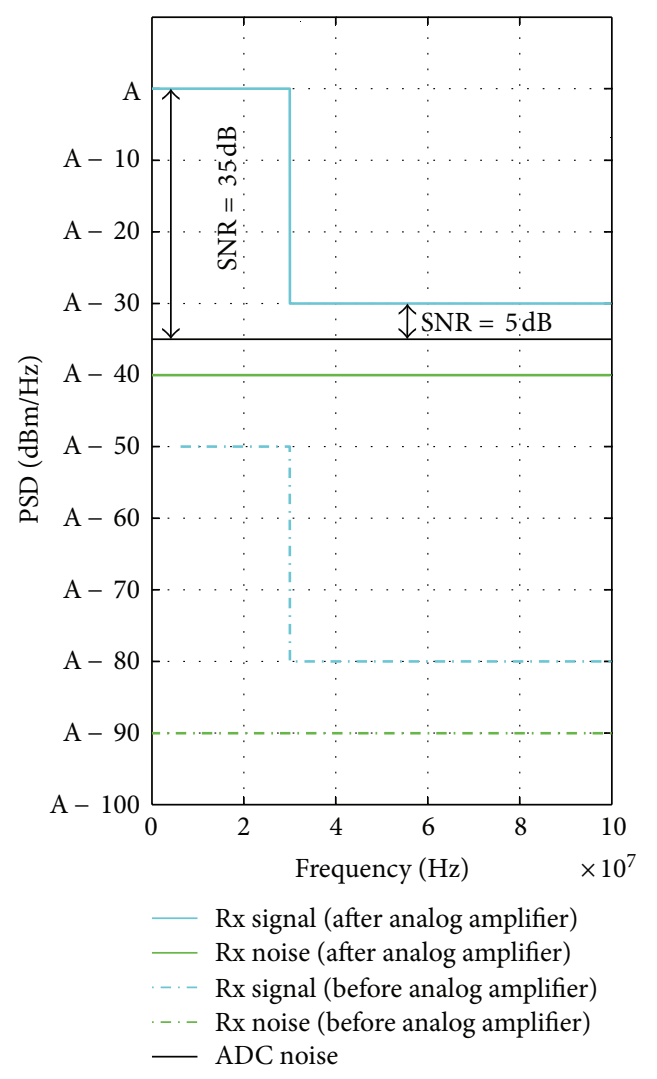

(a)

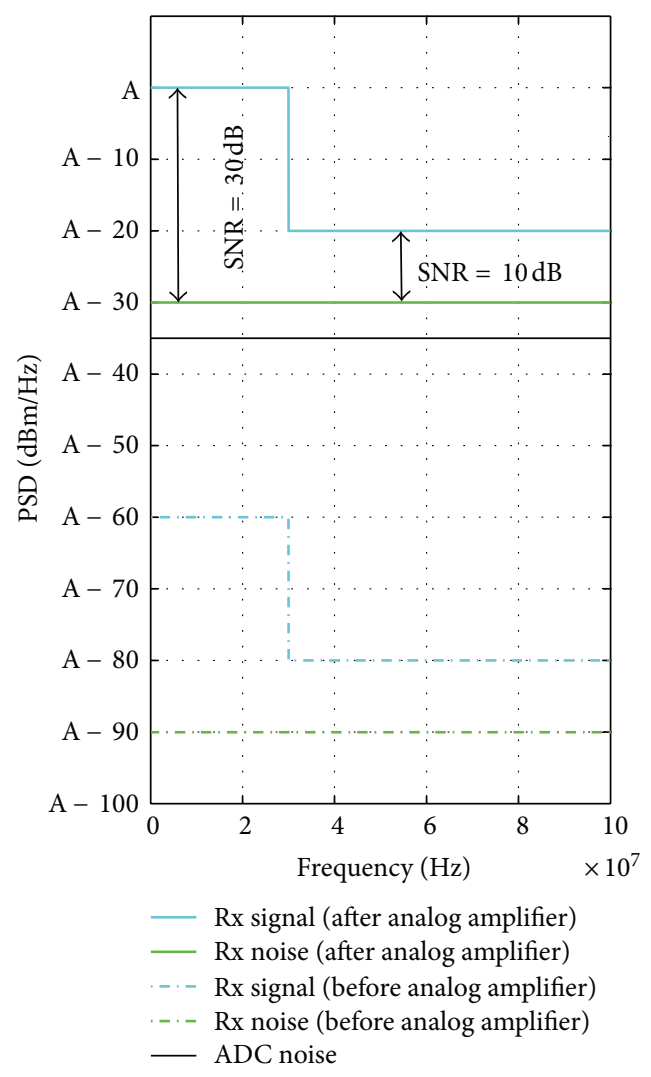

(b)

Figure 14: Benefits of power back-off. (a) No power back-off, (b) $10 \mathrm{~dB}$ power back-off.

in the $1.8-30 \mathrm{MHz}$ frequency band so that it is only $20 \mathrm{~dB}$ greater compared to the $1.8-86 \mathrm{MHz}$ band. Again, the analog amplifier brings the received signal to the A level. However in this case, the dominating noise is no longer the ADC noise and the obtained SNRs are $30 \mathrm{~dB}$ and $10 \mathrm{~dB}$ in the $1.8-30 \mathrm{MHz}$ and $30-86 \mathrm{MHz}$, respectively.

In this example, the power back-off technique results in a $5 \mathrm{~dB}$ SNR reduction for the carriers in the lower frequency band, and a $5 \mathrm{~dB}$ SNR increase for the carriers in the upper frequency band. Given the larger bandwidth of the upper frequency band there will be an overall throughput gain due to transmit power back-off.

Transmit power back-off is also an effective interference mitigation technique. For instance, in Europe, the ability of a PLC transmitter to reduce the transmit power depending on the attenuation link is a possible requirement considered in [32], though the procedure described in [32] does not consider Quality of Service (QoS) requirements of PLC modems.

3.4.2. EMC Friendly Power Boost Using $S_{11}$ Parameter. The EMC Friendly Power Boost is a mechanism introduced in the HomePlug AV2 specification to optimize the transmit power by monitoring the input port reflection coefficient at the transmitting modem. This coefficient is known as the
$S_{11}$ parameter. PSD limits as proposed in the HomePlug AV2 specification are based on representative statistics of the impedance match at the interface between the device port and the power line network. In practice, the input impedance of the power line network is frequency selective and varies for different network configurations. This leads to part of the transmit power being dissipated within the transmitter. The input port reflection coefficient, or input return loss, is characterized by the $S$-parameter $S_{11}$, and the part of transmit power dissipated at the transmitter is given by the amount $20 \cdot \log 10\left(\left|S_{11}\right|\right)$ in $\mathrm{dB}$. In situations where the impedance mismatch is large (and thus the $S_{11}$ parameter is large), only a small part of the input power is effectively transferred to the power lines. In those cases, the Electromagnetic Interference (EMI) induced by the PLC modem is reduced and can be much lower than the values recommended by EMC regulation limits.

In order to compensate for the frequency selective impedance mismatch at the interface between the device port and the power line network, HomePlug AV2 modems adapt their transmission mask, upon measurement of the $S_{11}$ parameter at the transmitter. The transmitted signal power is increased by an Impedance Mismatch Compensation (IMC) factor, leading a more effective power transmission to the power line medium. While increasing the Tx power leads to 
an increase of the radiated EMI, the design of the IMC factor ensures that the resulting EMI continuously falls below the targeted EMC regulation limits.

A statistical analysis was conducted on the practical values of the $S_{11}$ parameter and the effectiveness of the EMC Friendly Power Boost technique based on a series of measurements performed by the ETSI Specialist Task Force $410[6-10,33]$. $S_{11}$ parameter and EMI measurements were taken over the $1 \mathrm{MHz}-100 \mathrm{MHz}$ range in 6 countries: Germany, Switzerland, Belgium, UK, France, and Spain. The modem used for measurements is described in [6-8].

The $S_{11}$ measurements considered in this study consist of 3 differential feeding possibilities (L-N, N-PE, and PE-L), and 1 common mode feeding (CM). For the EMI measurements, we consider the measurements taken with different feeding possibilities, that is, differential feeding with the other differential ports being unterminated or terminated with $50 \mathrm{Ohm}$, and CM feeding.

The measurement set used in this analysis consists of 478 frequency sweeps, that can be categorized as follows:

(i) 6 different locations in Germany and 3 different locations in France.

(ii) 264 measurements outdoor at $10 \mathrm{~m}, 43$ measurements outdoor at $3 \mathrm{~m}$, and 171 measurements indoors.

Statistical analysis of this experimental data allowed designing the practical implementation of the EMC Friendly Power Boost technique. In the following, we define the Impedance Mismatch Compensation (IMC) factor as (in $\mathrm{dB}$ )

$$
\begin{array}{r}
\operatorname{IMC}(k)=\min \left(\operatorname { m a x } \left(10 \cdot \log _{10}\left(\frac{1}{1-\left|S_{11}(k)\right|^{2}}\right)\right.\right. \\
\left.-M(k), 0), \mathrm{IMC}_{\max }\right),
\end{array}
$$

where $S_{11}(k)$ is the carrier dependent estimate of the $S_{11}$ parameter, $M$ is a margin accounting for possible estimate uncertainties in the measurement of parameter $S_{11}$, and $\mathrm{IMC}_{\max }$ is the maximum allowed value of the IMC factor in dB.

Figures 15 and 16 represent the statistical CDF of the $S_{11}$ parameter as well as the corresponding $\mathrm{IMC}_{0}$ factor, computed with a margin $M$ of $0 \mathrm{~dB}$. These statistics are based on the experimental data collected during the ETSI STF 410 measurement campaign.

On Figure 15, one can read that the $S_{11}$ parameter is larger than $-10 \mathrm{~dB}$ for $80 \%$ of the cases. This means that for $80 \%$ of the records, more than $10 \%$ of the energy is reflected back towards the transmitter.

More interestingly, Figure 16 gives an idea of the potential power increase offered by the EMC Friendly Power Boost technique.

(i) For $40 \%$ of the records, the $\mathrm{Tx}$ power could be increased by more than $2 \mathrm{~dB}$ to compensate for the impedance mismatch.

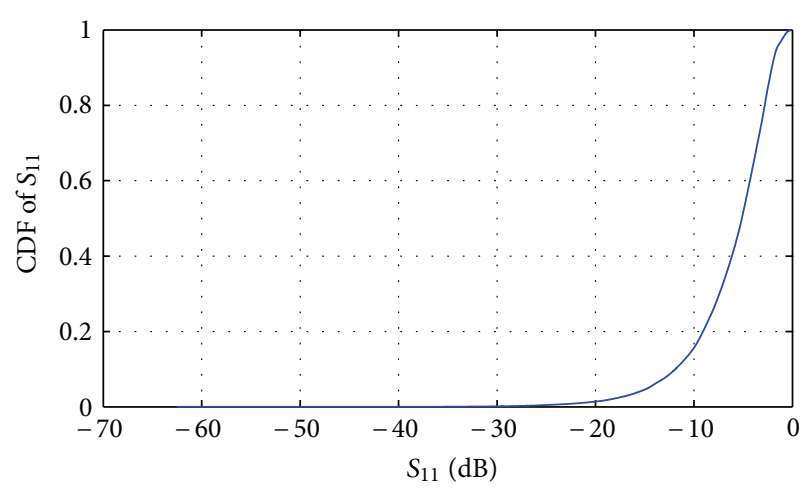

FIGURE 15: CDF of $S_{11}$ parameter.

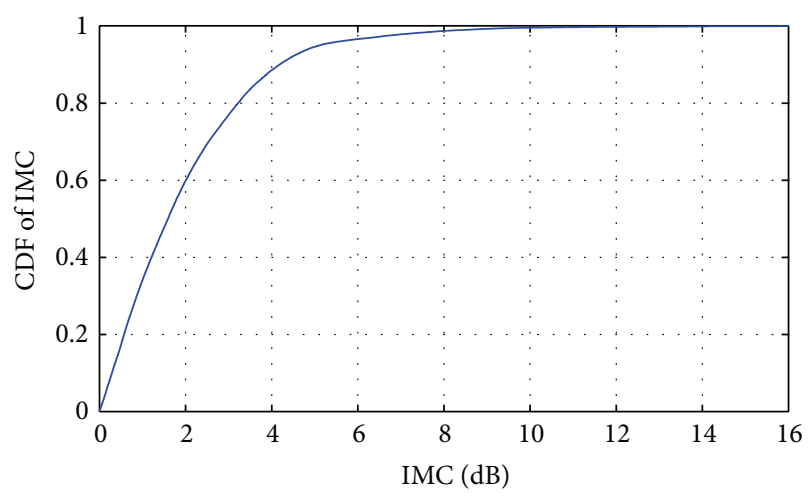

FIGURE 16: CDF of IMC parameter.

(ii) For $10 \%$ of the records, the Tx power could be increased by more than $4 \mathrm{~dB}$ to compensate for the impedance mismatch.

We then focused on the effect of the EMC Friendly Power Boost technique on the radiated EMI statistics. The recorded values allowed the computation of two statistics:

(i) the statistical CDF of the recorded EMI in terms of E-field for all frequencies and feeding possibilities without applying any power boost,

(ii) the statistical CDF of the recorded EMI in terms of Efield for all frequencies and feeding possibilities when applying the EMC Friendly Power Boost.

Figure 17 presents, for each percentile of the CDF, the difference in $\mathrm{dB}$ between the E-Field CDFs for the two methods of transmission.

Different observations can be made from this figure. First, the application of the EMC Friendly Power Boost leads to an increase of the radiated field CDF comprised between 0 and $6 \mathrm{~dB}$. Note that the extreme value of $6 \mathrm{~dB}$ arises for one of the lowest values of radiated field, and, hence, is not relevant. Secondly, in general, the application of the EMC Friendly Power Boost increases the radiated power CDF by about $2 \mathrm{~dB}$. More importantly, the increase of the radiated power CDF is lower than $2 \mathrm{~dB}$ for the $25 \%$ most radiating cases. This practically means that in the worst case scenarios where the 


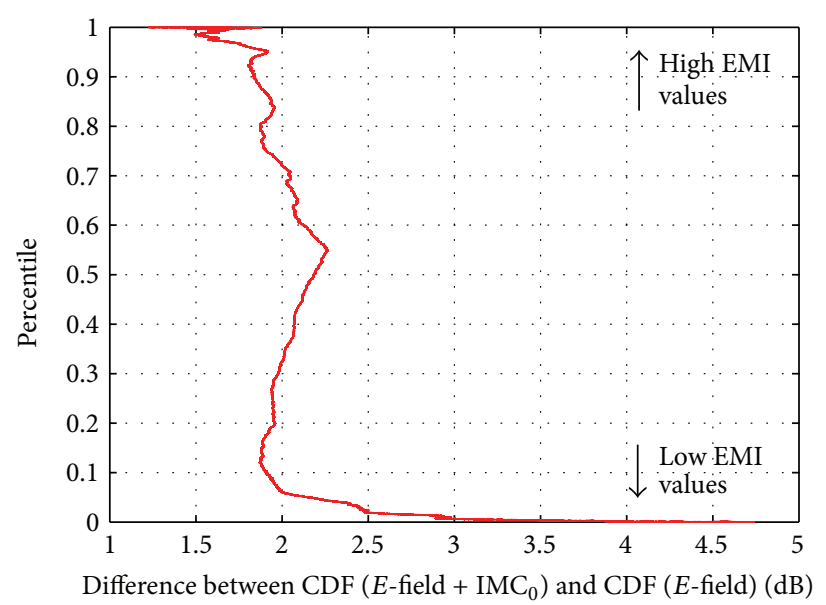

FIGURE 17: Difference in dB between the CDFs of the radiated EMI before and after applying the EMC Friendly Power Boost.

modems produce the largest EMI, the application of the IMC factor does not increase the EMI by more than $2 \mathrm{~dB}$. This value can be compared with the CDF of the IMC factor given in Figure 16. Although the IMC factor is larger than $2 \mathrm{~dB}$ in $40 \%$ of the cases and larger than $4 \mathrm{~dB}$ in $10 \%$ of the cases, the application of the EMC Friendly Power Boost techniques does not increase the radiated power CDF extreme values by more than $2 \mathrm{~dB}$. Of course, even an increase of the EMI by $2 \mathrm{~dB}$ is not acceptable. Therefore, a margin $M$ of $2 \mathrm{~dB}$ is applied when increasing the Tx power using the IMC factor.

Based on this study, we conclude that the application of the EMC Friendly Power Boost technique provides a significant gain in terms of transmit power increase for a large number of configurations, where the impedance mismatch causes the dissipation of the signal at the transmitter. In addition, the statistical analysis shows that this technique will not lead to an increase of the undesirable radiated interference, in particular in the worst EMI scenarios, as long as a margin $M$ of $2 \mathrm{~dB}$ is used in the computation of the IMC factor, as specified in (11). Finally, a recommended limit for the maximum allowed value of the IMC factor is $\mathrm{IMC}_{\max }=$ $6 \mathrm{~dB}$.

3.5. Additional PHY Improvements. In addition to the MIMO technology, the frequency band extension, the Efficient Notching, and power optimization techniques such as the power back-off and the EMC friendly power boost, other elements of the PHY layer were modified as presented in the paragraphs below.

3.5.1. New Time Domain Parameters. In the HomePlug AV2 specification, a number of time domain parameters were refined. As the sampling frequency has increased from $75 \mathrm{MHz}$ to $200 \mathrm{MHz}$, the number of time samples for a given symbol duration is increased by a factor 8/3; the IFFT interval is 8192 samples in length, and the number of samples in the HomePlug AV Guard Interval has increased accordingly. In addition, new features have been added: (i) The Transition Interval defines the part of the Rolloff Interval dedicated to the transition window, allowing more flexibility in the choice of the window (see Section 3.3)

(ii) A new Guard Interval has been defined for the HomePlug AV2 Short Delimiter (see Section 4.2.1)

(iii) The payload symbol Guard Interval has been made variable and can be as short as $0 \mu \mathrm{s}$. It can also be increased up to $19.56 \mu \mathrm{s}$. This allows adaptation to a wide range of channel conditions and removes the overhead of the Guard Interval for channels that have either very low multipath dispersion, or that are completely limited by the receive noise and not by ISI.

3.5.2. Additional Constellations. In HomePlug AV, the maximum constellation size is 1024-QAM, corresponding to 10 coded bits per carrier. HomePlug AV2 also provides support for 4096-QAM, which corresponds to 12 bits per carrier. The higher constellation size increases the peak PHY rates by $20 \%$. Practically, the increased throughput will be available mostly on average to very good channels, but even some of the poorer channels sometimes have frequency bands in which high SNRs can be achieved, and the increased constellation size can be used.

3.5.3. Forward Error Correction (FEC) Coding. HomePlug AV2 uses the same duobinary Turbo Code as HomePlug AV. In addition to the code rates of $1 / 2$ and $16 / 21$, HomePlug AV2 also provides support for a 16/18 code rate. This allows more granularity in the compromise between robustness and throughput degradation. For this new code rate, a new puncturing structure is defined, as well as a new channel interleaver. In addition, a new Physical Block size of 32 octets is defined, which includes specification of a new termination matrix for the FEC as well as a new interleaver seed table. The 32-byte octet PBs are used in the PHY level acknowledgements and allow for the acknowledgement of much larger packet sizes that are supported with the increased PHY rates possible in HomePlug AV2.

3.5.4. Line Cycle Synchronization. The HomePlug AV2 specification describes also the device operation in scenarios where there is no alternating current (AC) line cycle (e.g., a direct current (DC) power line) or when the AC line cycle is different from $50 \mathrm{~Hz}$ or $60 \mathrm{~Hz}$. In this case, the Central Coordinator is preconfigured to select a Beacon Period matching either $50 \mathrm{~Hz}$ (i.e., Beacon Period is $40 \mathrm{msec}$ ) or $60 \mathrm{~Hz}$ (i.e., Beacon Period is $33.33 \mathrm{msec}$ ). One key use case where this feature is useful is the transfer of data towards a multimedia equipped electrical vehicle during the electrical charging phase (using DC power).

\section{MAC Layer Improvements of HomePlug AV2}

4.1. Power Save Modes. HomePlug AV2 stations improve their energy efficiency in standby mode through the adoption 
of the specific Power Save Mode already defined in the HomePlug Green PHY [4] specification. In Power Save mode stations reduce their average power consumption by periodically transitioning between Awake and Sleep states. Stations in the Awake state can transmit and receive packets over the power line. In contrast, stations in Sleep state temporarily suspend transmission and reception of packets over the power line.

We introduce some basic terms useful to describe the Power Save Mode.

(i) Awake Window: period of time during which the station is capable of transmitting and receiving frames. The Awake Window has a range from a few milliseconds to several Beacon Periods (a Beacon Period is two times the AC line cycle: $40 \mathrm{~ms}$ for a $50 \mathrm{~Hz}$ AC line and $33.3 \mathrm{~ms}$ for a $60 \mathrm{~Hz} \mathrm{AC}$ ).

(ii) Sleep Window: period of time during which the station is not capable of transmitting or receiving frames.

(iii) Power Save Period (PSP): interval from the beginning of one Awake Window to the beginning of the next Awake Window. Power Save Period is restricted to $2 k$ multiples of Beacon Periods (i.e., 1 Beacon Period, 2 Beacon Periods, 4 Beacon Periods, ...).

(iv) Power Save Schedule (PSS): the combination of the values of the PSP and of the Awake Widow duration. To communicate with a station in Power Save mode, other stations in the logical network (AVLN) need to know its PSS.

Potentially, the specification allows aggressive PSSs constituted by an Awake Window duration of $1.5 \mathrm{~ms}$ and a PSP of 1024 Beacon Periods, that will cause over 99\% energy saving compared to HomePlug AV. In practice, some in-home applications will require lower latency and response time, and a balance will take place reducing the mentioned gain. This is particular appealing for applications that foresee a PLC utilization that is variable during the day (for instance large utilization in daylight time and small utilization during night period). It is worth highlighting that the HomePlug AV2 specification is flexible in allowing each station in a network to have a different PSS. Given these remarks, in order to enable efficient Power Save operation without causing difficulties to regular communication, all the stations in a network need to know the PSSs of the other stations. The network Central Coordinator (CCo) has a key role since it grants the requests of the different stations to enter and exit from Power Save mode operation. Moreover, it distributes the different PSSs to all the stations in the network. When needed a CCo can

(i) optionally disable Power Save mode for all stations of the AVLN,

(ii) optionally wake up a station in Power Save mode.

The shared knowledge of the PSS allows stations communicating during the common Awake Windows (the HomePlug AV2 and HomePlug Green PHY specifications have

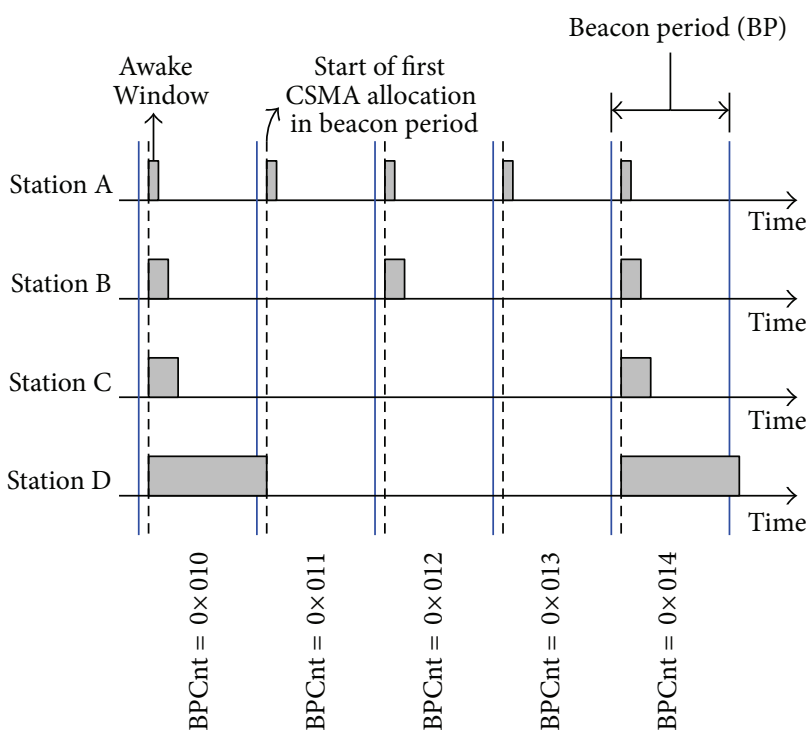

Figure 18: Example of Power Save operation in HomePlug AV2 and HomePlug Green PHY.

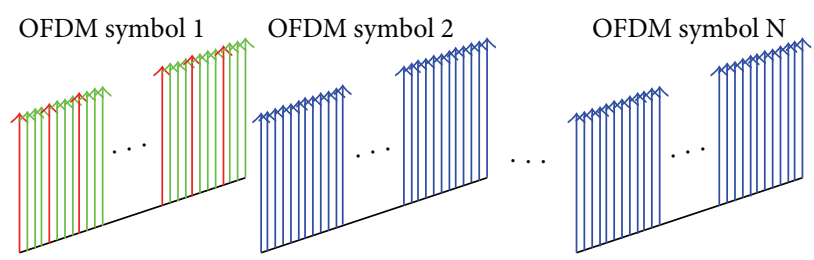

\footnotetext{
$\uparrow$ Preamble Carrier

$\uparrow$ Frame Control Carrier

$\uparrow$ Data Carrier
}

FIGURE 19: Short Delimiter.

structured the protocol insuring that at least one superposition of all the Awake Windows occurs). This overlap interval can also be used for transmission of information that needs to be received by all stations within the AVLN.

Figure 18 shows an example of Power Save Schedule of the four stations $\{\mathrm{A}, \mathrm{B}, \mathrm{C}$, and $\mathrm{D}\}$. All stations save more than $75 \%$ energy compared to HomePlug AV. Note that in this example, stations A and B can communicate once every 2 Beacon Periods. Moreover, all the stations are always awake at the same time once every 4 Beacon Periods thus preserving communication possibility.

4.2. Short Delimiter and Delayed Acknowledgement. The Short Delimiter and Delayed Acknowledgement features were added to HomePlug AV2 to improve efficiency by reducing the overhead associated with transmitting payloads over the power line channel. In HomePlug AV, this overhead results in relatively poor efficiency for transmission control protocol (TCP) payloads. One goal that was achieved with 


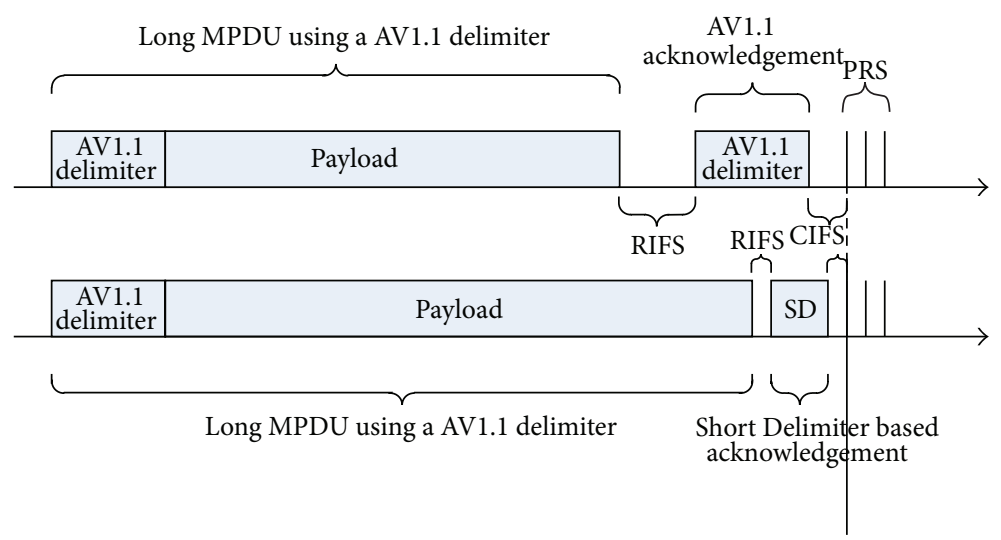

FIGURE 20: Short delimiter efficiency improvement.

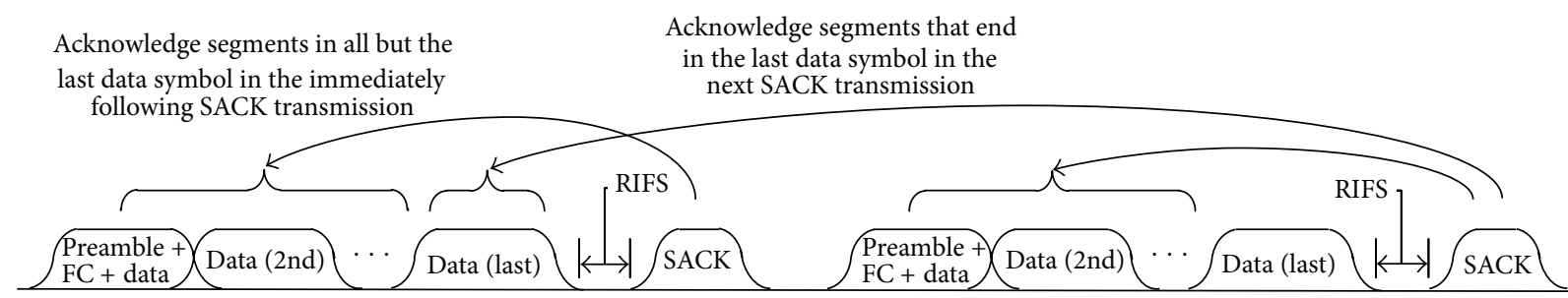

FIGURE 21: Delayed Acknowledgement.

these new features was TCP efficiency that improved to be relatively close to that of UDP.

In order to send a packet carrying payload data over a noisy channel, signaling is required for a receiver to detect the beginning of the packet and to estimate the channel so that the payload can be decoded, and additional signaling is needed to acknowledge the payload was received successfully. Interframe spaces are also required between the payload transmission and the acknowledgement for the processing time at the receiver to decode and check the payload for accurate reception and to encode the acknowledgement. This overhead is even more significant for TCP payload since the TCP acknowledgement payload must be transmitted in the reverse direction.

4.2.1. Short Delimiter. The delimiter specified in HomePlug AV contains the Preamble and Frame Control symbols and is used for the beginning of data PPDUs as well as for immediate acknowledgements. The length of the HomePlug AV delimiter is $110.5 \mu \mathrm{s}$ and can represent a significant amount of overhead for each channel access. A new single OFDM symbol delimiter is specified in HomePlug AV2 to reduce the overhead associated with delimiters by reducing the length to $55.5 \mu \mathrm{s}$. Figure 19 shows that every fourth carrier in the first OFDM symbol is assigned as a Preamble Carrier, and the remaining carriers encode the Frame Control. The following OFDM symbols encode data the same as in HomePlug AV.

Figure 20 demonstrates the efficiency improvement when the HomePlug AV2 Short Delimiter is used for the acknowledgement of a CSMA Long MPDU (MAC Protocol Data Unit) compared to the HomePlug AV delimiter. Not only is the length of the delimiter reduced from 110.5 to $55.5 \mu \mathrm{s}$, the Response Inter-Frame Space (RIFS) and Contention InterFrame Space (CIFS) can also be reduced to $5 \mu \mathrm{s}$ and $10 \mu \mathrm{s}$, respectively. Reduction of RIFS requires Delayed Acknowledgement, which is described in Section 4.2.2. Backward compatibility when contending with HomePlug AV devices is maintained by indicating the same length field for virtual carrier sense in both cases so that the position of the priority resolution symbols (PRS) contention remains the same. A field in the Frame Control of the Long MPDU indicates the Short Delimiter format to a HomePlug AV2 device so that it can correctly determine the length of the payload.

4.2.2. Delayed Acknowledgement. The processing time to decode the last OFDM symbol and encode the acknowledgement can be quite high, thus requiring a rather large Response Inter-Frame Space (RIFS). In HomePlug AV, since the preamble is a fixed signal, the preamble portions of the acknowledgement can be transmitted while the receiver is still decoding the last OFDM symbol and encoding the payload for the acknowledgement. With the Short Delimiter, the preamble is encoded in the same OFDM symbol as the Frame Control for the acknowledgement, so the RIFS would need to be larger than for HomePlug AV, eliminating much of the gain the Short Delimiter provides.

Delayed Acknowledgement solves this problem by acknowledging the segments ending in the last OFDM symbol in the acknowledgement transmission of the next PPDU, as shown in Figure 21. This permits practical implementations with a very small RIFS, reducing the RIFS overhead close to zero. HomePlug AV2 also allows the 


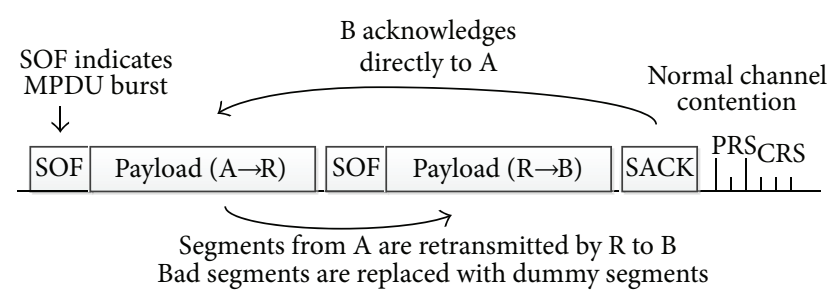

FIGURE 22: Immediate repeating channel access for CSMA.

option of delaying acknowledgement for segments ending in the second to last OFDM symbol to provide flexibility for implementers.

4.3. Immediate Repeating. HomePlug AV2 supports repeating and routing of traffic to not only handle hidden nodes but also to improve coverage (i.e., performance on the worst channels).

With HomePlug AV2, hidden nodes are extremely rare. However, some links may not support the data rate required for some applications such as a $3 \mathrm{D} \mathrm{HD}$ video stream. In a network where there are multiple HomePlug AV2 devices, the connection through a repeater typically provides a higher data rate than the direct path for the poorest $5 \%$ of channels.

Immediate Repeating is a new feature in HomePlug AV2 that enables high efficient repeating. Immediate Repeating provides a mechanism to use a repeater with a single channel access, and the acknowledgement does not involve the repeater. As shown in Figure 22, station A transmits to the repeater $R$. In the same channel access, repeater $R$ transmits all payload received from station $A$ to station $B$. B sends an acknowledgement directly to A. With this approach, latency is actually reduced with repeating, assuming the resulting data rate is higher, the obvious criteria for using repeating in the first place. Also, resources required by the repeater are minimized since the repeater uses and immediately frees memory it would require for receiving payload destined for it. Also the receiver has no retransmission responsibility for failed segments.

\section{Coexistence with Other PLC Technologies}

5.1. Inter System Protocol. Intersystem Protocol (ISP) allows coexistence between noninteroperable devices sharing the same power line medium. Using the current ISP protocol, noninteroperable devices are able to coexist. The HomePlug AV2 will operate in a 1901-FFT mode [5] in order to coexist with the other systems, in this case the TDM slot allocated for the 1901-FFT is to be used by the HomePlug AV2 system.

The ISP protocol allows a TDM scheme to be implemented between coexisting in-home systems and between coexisting in-home and access systems. Each of the PLC system categories is allocated a particular ISP window in a round-robin fashion. The allocation is determined by (1) the number of systems on the power line, (2) the type of the systems present, and (3) the access system bandwidth request, as defined in the 1901 standard [34].
The TDM synchronization period for the in-home and access systems is defined with the parameter $T_{H}$ in Figure 23. There are four ISP windows within a single $T_{H}$ period. Each ISP window is further divided into three TDM units (TDMU), so there are a total of twelve TDMUs in each $T_{H}$ period, labeled TDMU\#0 through TDMU\#11. Each TDMU is further divided into eight TDM slots (TDMS), labeled TDMS\#0 through TDMS\#7.

Figure 23 illustrates the TDM partitioning relative to the AC line cycles. The ISP window is used to generate and detect the ISP signal that is allocated within the first TDMS\#0 in TMDU\#0, TMDU\#3, TMDU\#6, and TDMU\#9. The purpose and nature of the ISP signals will be described later. Note: the term Beacon Period in the HomePlug AV2 specification is similar to the $2 \mathrm{AC}$ line cycles of the TDMU.

5.2. ISP Signals. Coexistence signaling is carried out by the use of periodically repeating ISP signals within the ISP windows. The signals are used to convey information on coexisting system presence, resource requirements, and resynchronization request. Each PLC system category is allocated a particular ISP window in a round-robin fashion, as illustrated in Figure 23.

The ISP signal is transmitted using a range of designated phases that convey a range of information to be used by the system. This set of instantaneous information is termed the Network Status that defines the allocation of resources to each coexisting system.

The ISP signal is merely detected. By monitoring the ISP signal transmitted within the ISP windows allocated to other systems, a coexisting system is able to determine the number and type of coexisting systems present on the line and their resource requirements. Similarly, by monitoring the signal within its own ISP window, a coexisting system is able to detect a resynchronization request from one of the other coexisting systems.

The ISP signal consists of 16 consecutive OFDM symbols. Each OFDM symbol is formed by a set of "all-one" binary phase shift keying (BPSK), modulated onto the carrier waveforms using IFFT, and multiplied by a window function to reduce out-of-band energy complying with the transmit spectrum requirement. Since all devices send the signal simultaneously, the ISP signal must be sent with $8 \mathrm{~dB}$ less power than the normal transmission.

Timing parameter that is used for generating an ISP signal is described in Figure 24.

5.3. Startup and Resynchronization Procedures. The TDM synchronization scheme mentioned above is used such that each PLC system shares the medium without interfering with one another. However, it is possible that two or more systems are synchronized to two or more different, mutually visible ISP sequences [35]. In such cases, in order to prevent mutual interference, it is important that they resynchronize to the same ISP sequence.

In other words, whenever a HomePlug AV2 device starts up or restarts, it needs to be aware of the presence of any other systems with which it is able to coexist. Accordingly, a startup 

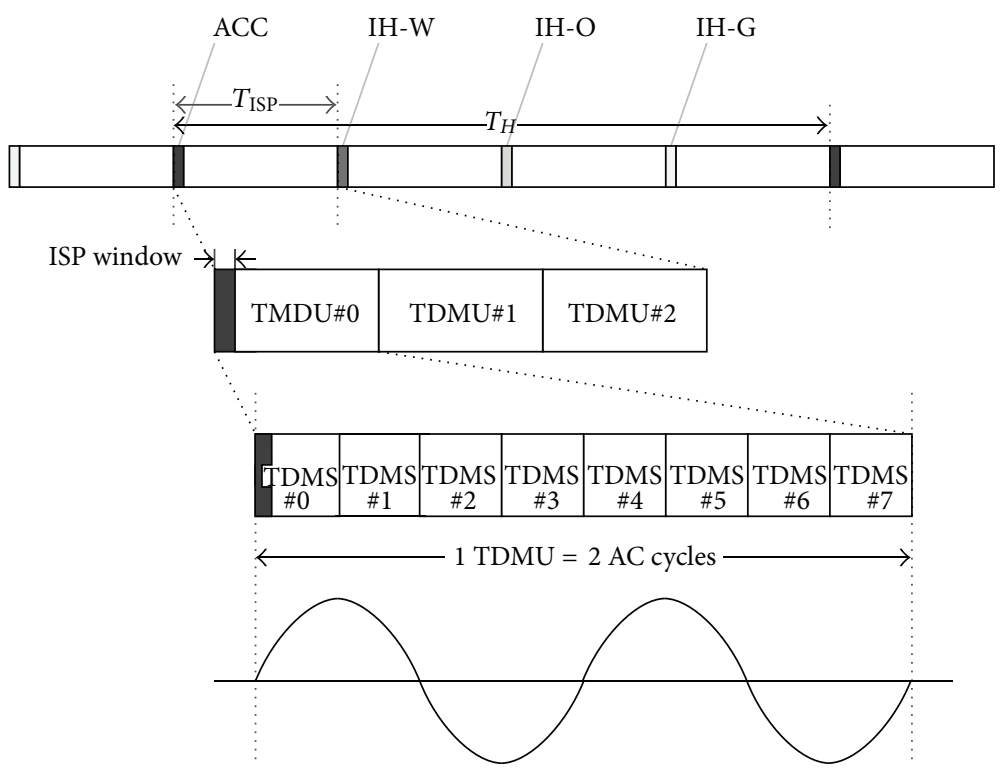

Figure 23: Time Division Multiplexing on ISP.

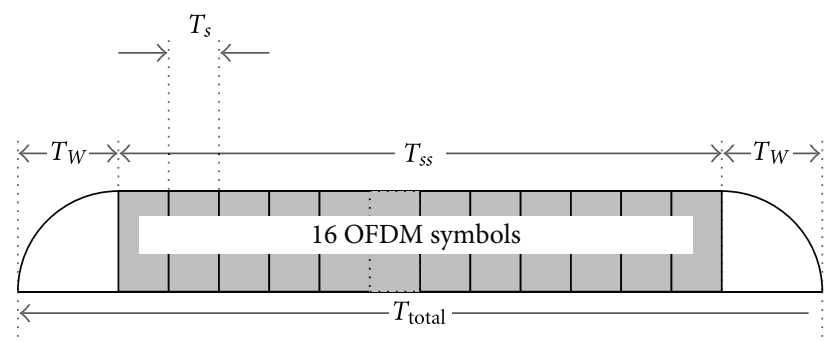

\begin{tabular}{|c|c|c|}
\hline $\begin{array}{c}\text { Timing } \\
\text { Parameters }\end{array}$ & Description & Time $(\mu \mathrm{s})$ \\
\hline$T_{s}$ & IFFT interval & 5.12 \\
\hline$T_{s s}$ & OFDM symbols duration & $\begin{array}{c}T_{\text {total }}-2 \times \\
T_{W}\end{array}$ \\
\hline$T_{W}$ & Windowing duration & $\leq 10.24$ \\
\hline$T_{\text {total }}$ & ISP signal interval & 81.92 \\
\hline
\end{tabular}

Figure 24: ISP Signal Timing Parameter.

and resynchronization procedure is defined within the ISP protocol that allows the starting system to synchronize with an existing system.

\section{Gain of HomePlug AV2 Compared to HomePlug AV}

The AV TWG has evaluated the performance of the HomePlug AV2 specification; this activity has been fundamental in order to see if the produced specification meets the requirements of all the stakeholders. The following tables show the performance improvement as compared to HomePlug AV in terms of coverage. These preliminary results are based on a 6-home field test in Florida, home sizes 1900-3300 sq. ft.

Table 1 presents the results in a 2-node network scenario; $95 \%$ of nodes experiment a throughput improvement greater
TABLE 1: Improvement of HomePlug AV2 in a 2-node network.

\begin{tabular}{lc}
\hline $\begin{array}{l}\text { Coverage based on UDP } \\
\text { throughput }\end{array}$ & $\begin{array}{c}\text { Percentage of throughput } \\
\text { improvement of HomePlug AV2 } \\
\text { compared to HomePlug AV }\end{array}$ \\
\hline $95 \%$ & $>136 \%$ \\
$5 \%$ & $>220 \%$ \\
\hline
\end{tabular}

TABLE 2: Improvement of HomePlug AV2 in a 4-node network.

\begin{tabular}{lc}
\hline $\begin{array}{l}\text { Coverage based on UDP } \\
\text { throughput }\end{array}$ & $\begin{array}{c}\text { Percentage of throughput } \\
\text { improvement of HomePlug AV2 } \\
\text { compared to HomePlug AV }\end{array}$ \\
\hline $99 \%$ & $>131 \%$ \\
$5 \%$ & $>173 \%$ \\
\hline
\end{tabular}

than $136 \%$ compared to HomePlug AV (which means a performance enhancement by a factor nearly equal to 2.4!). Benefits are even higher when considering the most favorable connections (see the improvement at the 5\% coverage value). Table 2 considers a 4 -node scenario where 3 streams carrying different data are transmitted from one source (e.g., a SetTop Box) to 3 different destinations (e.g., TVs). In this case, the improvement in the aggregate throughput is relevant for the $99 \%$ of networks compared to HomePlug AV (more than $131 \%)$.

Note that the benefits of the HomePlug AV2 technology are expected to be greater (which explains the ">" symbol) than the ones shown in Tables 1 and 2 since, for instance, a $2 \times 2$ MIMO was tested in Florida; $2 \times 3$ or $2 \times 4$ MIMO would likely provide better performance.

Another interesting figure is the theoretical maximum PHY throughput for the system, for different options of the standard (Table 3 ). This number represents the throughput of transmitted bits on the PHY layer for optimum channel 
TABLE 3: Maximum PHY rate computation.

\begin{tabular}{lc}
\hline $\begin{array}{l}\text { System configuration } \\
\text { (North American tone mask) }\end{array}$ & Max PHY rate \\
\hline $\begin{array}{l}\text { HomePlug AV (1.8-30 MHz) } \\
(917 \text { carriers, } 10 \text { bits/carr, } 5.56 \mu \mathrm{s} \mathrm{GI})\end{array}$ & $197 \mathrm{Mbps}$ \\
\hline $\begin{array}{l}\text { IEEE } 1901 \text { (1.8-50 MHz) } \\
(1974 \text { carriers, } 12 \text { bits/carr, } 1.6 \mu \mathrm{s} \mathrm{GI})\end{array}$ & $556 \mathrm{Mbps}$ \\
\hline $\begin{array}{l}\text { HomePlug AV2 SISO (1.8-86.13 MHz) } \\
(3455 \text { carriers, } 12 \text { bits/carr, } 0.0 \mu \mathrm{s} \mathrm{GI})\end{array}$ & $1012 \mathrm{Mbps}$ \\
\hline $\begin{array}{l}\text { HomePlug AV2 MIMO }(1.8-86.13 \mathrm{MHz}) \\
(3455 \text { carriers, } 12 \text { bits/carr, } 0.0 \mu \mathrm{s} \mathrm{GI}, 2 \text { streams })\end{array}$ & $2024 \mathrm{Mbps}$ \\
\hline
\end{tabular}

conditions and gives an idea of the benefits of different features. It can be seen that if the full frequency range is used, HomePlug AV2 provides a 1 Gbps throughput in SISO configuration and $2 \mathrm{Gbps}$ in a MIMO configuration.

\section{Conclusion}

In this paper, an overview of HomePlug AV2 has been presented. The overall system architecture and the key technical HomePlug AV2 improvements introduced at PHY and MAC layers have been described. It has been also shown the related performance improvements were achieved by HomePlug AV2 while ensuring both backward compatibility versus HomePlug AV and the coexistence with other power line technologies.

The HomePlug AV2 performance presented in this work has been assessed by AV TWG through simulations based on field measurements.

The results show the significant benefits introduced by the new set of HomePlug AV2 features, both in terms of achievable data rate and coverage.

\section{References}

[1] HomePlug Alliance, http://www.homeplug.org/.

[2] HomePlug Alliance, HomePlug AV White Paper, http://www .homeplug.org/.

[3] HomePlug Alliance, HomePlug AV Specification Version 2.0, January 2012.

[4] HomePlug Alliance, HomePlug GreenPHY, the Standard for In-Home Smart Grid Powerline Communications, http://www.homeplug.org/.

[5] IEEE Standard 1901-2010, IEEE Standard for Broadband over Power Line Networks: Medium Access Control and Physical Layer Specifications, http://standards.ieee.org/findstds/standard/1901-2010.html, 2010.

[6] ETSI TR 101 562, PowerLine Telecommunications (PLT); 19 MIMO PLT; Part 1: Measurement Methods of MIMO PLT, http://www.etsi.org/deliver/etsi_tr/101500_101599/10156201/ 01.03.01_60/tr_10156201v010301p.pdf, 2012.

[7] ETSI TR 101 562, PowerLine Telecommunications (PLT); MIMO PLT; Part 2: Setup and Statistical Results of MIMO PLT EMI Measurements, http://www.etsi.org/deliver/etsi_tr/ 101500_101599/10156202/01.02.01_60/tr_10156202v010201p.pdf, 2012.
[8] ETSI TR 101 562, PowerLine Telecommunications (PLT); MIMO PLT; Part 3: Setup and Statistical Results of MIMO PLT Channel and Noise Measurements, http://www.etsi.org/ deliver/etsi_tr/101500_101599/10156203/01.01.01_60/tr_10156203 v010101p.pdf, 2012.

[9] D. Schneider, A. Schwager, W. Bäschlin, and P. Pagani, "European MIMO PLC field measurements: channel analysis," in Proceedings of the IEEE International Symposium on Power Line Communications and its Applications (ISPLC '12), Beijing, China, March 2012.

[10] P. Pagani, R. Hashmat, A. Schwager, D. Schneider, and W. Bäschlin, "European MIMO PLC field measurements: noise analysis," in Proceedings of the IEEE International Symposium on Power Line Communications and its Applications (ISPLC '12), Beijing, China, March 2012.

[11] D. Veronesi, R. Riva, P. Bisaglia et al., "Characterization of inhome MIMO power line channels," in Proceedings of the IEEE International Symposium on Power Line Communications and Its Applications (ISPLC '11), pp. 42-47, Udine, Italy, April 2011.

[12] D. Rende, A. Nayagam, K. Afkhamie et al., "Noise correlation and its effect on capacity of inhome MIMO power line channels," in Proceedings of the IEEE International Symposium on Power Line Communications and Its Applications (ISPLC '11), pp. 60-65, Udine, Italy, April 2011.

[13] R. Hashmat, P. Pagani, A. Zeddam, and T. Chonavel, "MIMO communications for inhome PLC Networks: measurements and results up to $100 \mathrm{MHz}$," in Proceedings of the 14th IEEE International Symposium on Power Line Communications and its Applications (ISPLC '10), pp. 120-124, Rio de Janeiro, Brazil, March 2010.

[14] D. Schneider, Inhome power line communications using multiple input multiple output principles [Doctoral thesis], University of Stuttgart, Stuttgart, Germany, 2012.

[15] R. Hashmat, P. Pagani, A. Zeddam, and T. Chonavel, "A channel model for multiple input multiple output in-home power line networks," in Proceedings of the IEEE International Symposium on Power Line Communications and Its Applications (ISPLC '11), pp. 35-41, Udine, Italy, April 2011.

[16] R. Hashmat, P. Pagani, A. Zeddam, and T. Chonavel, "Analysis and modeling of background noise for inhome MIMO PLC channels," in Proceedings of the 14th IEEE International Symposium on Power Line Communications and its Applications (ISPLC '12), pp. 120-124, Beijing, China, March 2012.

[17] F. Versolatto and A. M. Tonello, "A MIMO PLC random channel generator and capacity analysis," in Proceedings of the IEEE International Symposium on Power Line Communications and Its Applications (ISPLC '11), pp. 66-71, Udine, Italy, April 2011.

[18] R. Hashmat, P. Pagani, T. Chonavel, and A. Zeddam, "A time domain model of background noise for In-home MIMO PLC networks," IEEE Transactions on Power Delivery, vol. 27, no. 4, pp. 2082-2089, 2012.

[19] A. Schwager, Powerline communications: significant technologies to become ready for integration [Doctoral thesis], University of Duisburg-Essen, Essen, Germany, 2010.

[20] A. Paulraj, R. Nabar, and D. Gore, Introduction To Space-Time Wireless Communications, Cambridge University Press, New York, NY, USA, 2003.

[21] L. Stadelmeier, D. Schneider, D. Schill, A. Schwager, and J. Speidel, "MIMO for inhome power line communications," in Proceedings of the 7th International ITG Conference on Source and Channel Coding (SCC '08), Ulm, Germany, January 2008. 
[22] A. Canova, N. Benvenuto, and P. Bisaglia, "Receivers for MIMO-PLC channels: throughput comparison," in Proceedings of the 14th IEEE International Symposium on Power Line Communications and its Applications (ISPLC '10), pp. 114-119, Rio de Janeiro, Brazil, March 2010.

[23] S. Katar, B. Mashburn, K. Afkhamie, H. Latchman, and R. Newman, "Channel adaptation based on cyclo-stationary noise characteristics in PLC systems," in Proceedings of the IEEE International Symposium on Power Line Communications and Its Applications (ISPLC '06), pp. 16-21, Orlando, Fla, USA, March 2006.

[24] A. M. Tonello, J. A. Cortes, and S. D’Alessandro, "Optimal time slot design in an OFDM-TDMA system over power-line time-variant channels," in Proceedings of the IEEE International Symposium on Power Line Communications and its Applications (ISPLC '09), pp. 41-46, Dresden, Germany, April 2009.

[25] A. Scaglione, P. Stoica, S. Barbarossa, G. B. Giannakis, and H. Sampath, "Optimal designs for space-time linear precoders and decoders," IEEE Transactions on Signal Processing, vol. 50, no. 5, pp. 1051-1064, 2002.

[26] A. R. Horn and R. C. Johnson, Matrix Analysis, Cambridge University Press, New York, NY, USA, 1985.

[27] A. Lozano, A. M. Tulino, and S. Verdú, "Optimum power allocation for parallel gaussian channels with arbitrary input distributions," IEEE Transactions on Information Theory, vol. 52, no. 7, pp. 3033-3051, 2006.

[28] S. D. 'Alessandro, A. M. Tonello, and L. Lampe, "Adaptive pulse-shaped OFDM with application to in-home power line communications," Telecommunication Systems Journal, vol. 51, no. 1, pp. 31-1311, 2011.

[29] F. J. Harris, "On the use of windows for harmonic analysis with the discrete Fourier transform," Proceedings of the IEEE, vol. 66, no. 1, pp. 51-83, 1978.

[30] K. H. Afkhamie, H. Latchman, L. Yonge, T. Davidson, and R. Newman, "Joint optimization of transmit pulse shaping, guard interval length, and receiver side narrow-band interference mitigation in the HomePlugAV OFDM system," in Proceedings of the IEEE 6th Workshop on Signal Processing Advances in Wireless Communications (SPAWC '05), pp. 996-1000, New York, NY, USA, June 2005.

[31] A. M. Tonello, S. D’Alessandro, and L. Lampe, "Cyclic prefix design and allocation in bit-loaded OFDM over power line communication channels," IEEE Transactions on Communications, vol. 58, no. 11, pp. 3265-3276, 2010.

[32] CENELEC, "Power line communication apparatus used in low voltage installations - radio disturbance characteristics-limits andmethods of measurement-part 1: apparatus for inhome use," Final Draft European Standard FprEN 50561-1, 2011.

[33] A. Schwager, W. Bäschlin, J. L. Gonzalez Moreno et al., "European MIMO PLC field measurements: overview of the ETSI STF410 campaign \& EMI analysis," in Proceedings of the IEEE International Symposium on Power Line Communications and its Applications (ISPLC '12), Beijing, China, March 2012.

[34] IEEE Standard 1901-2010, IEEE Standard for Broadband over Power Line Networks: Medium Access Control and Physical Layer Specifications, Chapter 16, "Inter System Protocol (ISP)", 2010.

[35] IEEE Standard 1901-2010, IEEE Standard for Broadband over Power Line Networks: Medium Access Control and Physical Layer Specifications, Annex R, "Resynchronization example", 2010. 

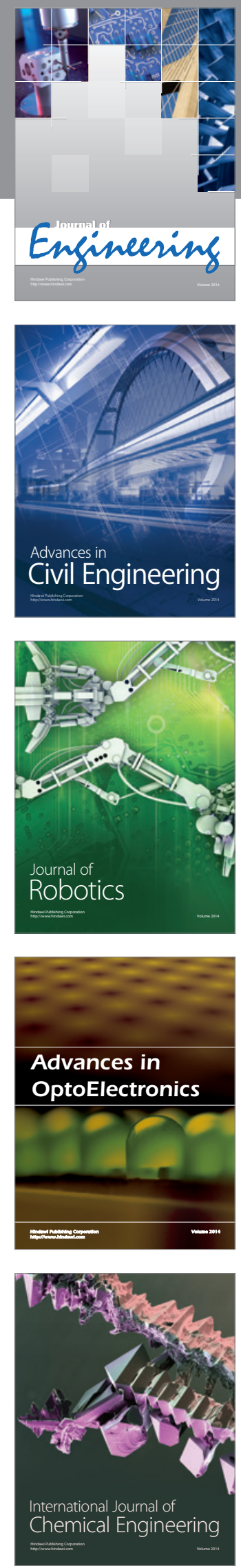

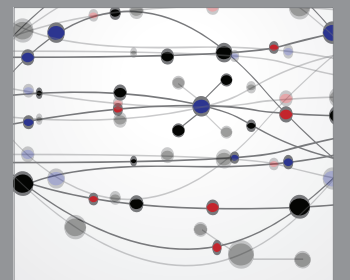

The Scientific World Journal
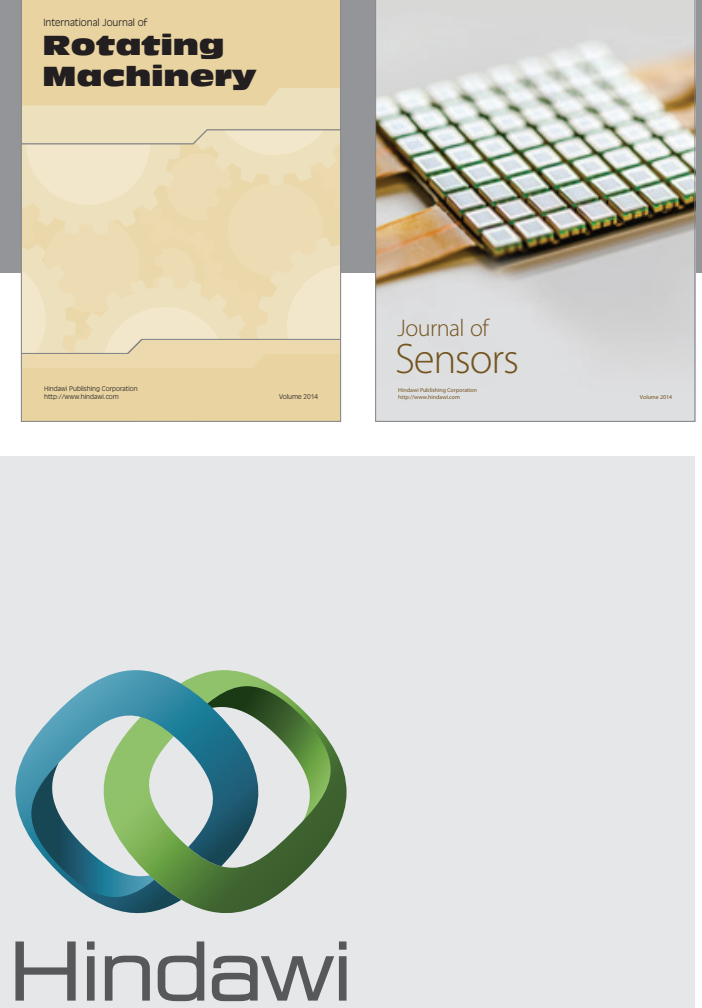

Submit your manuscripts at http://www.hindawi.com
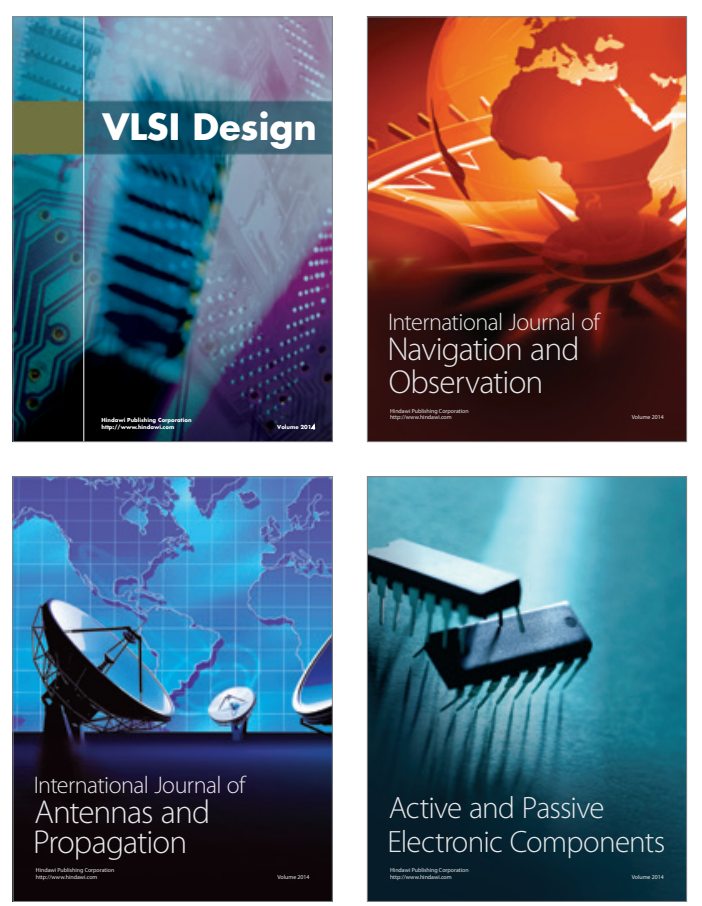
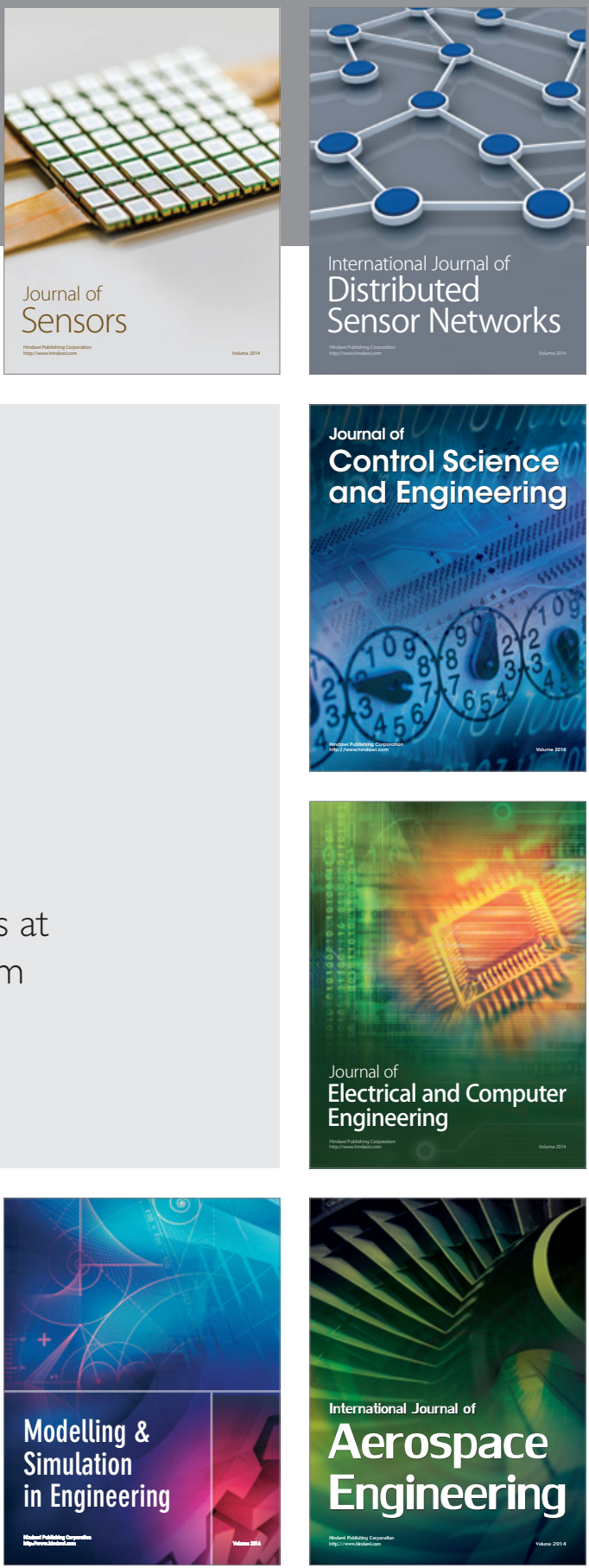

Journal of

Control Science

and Engineering
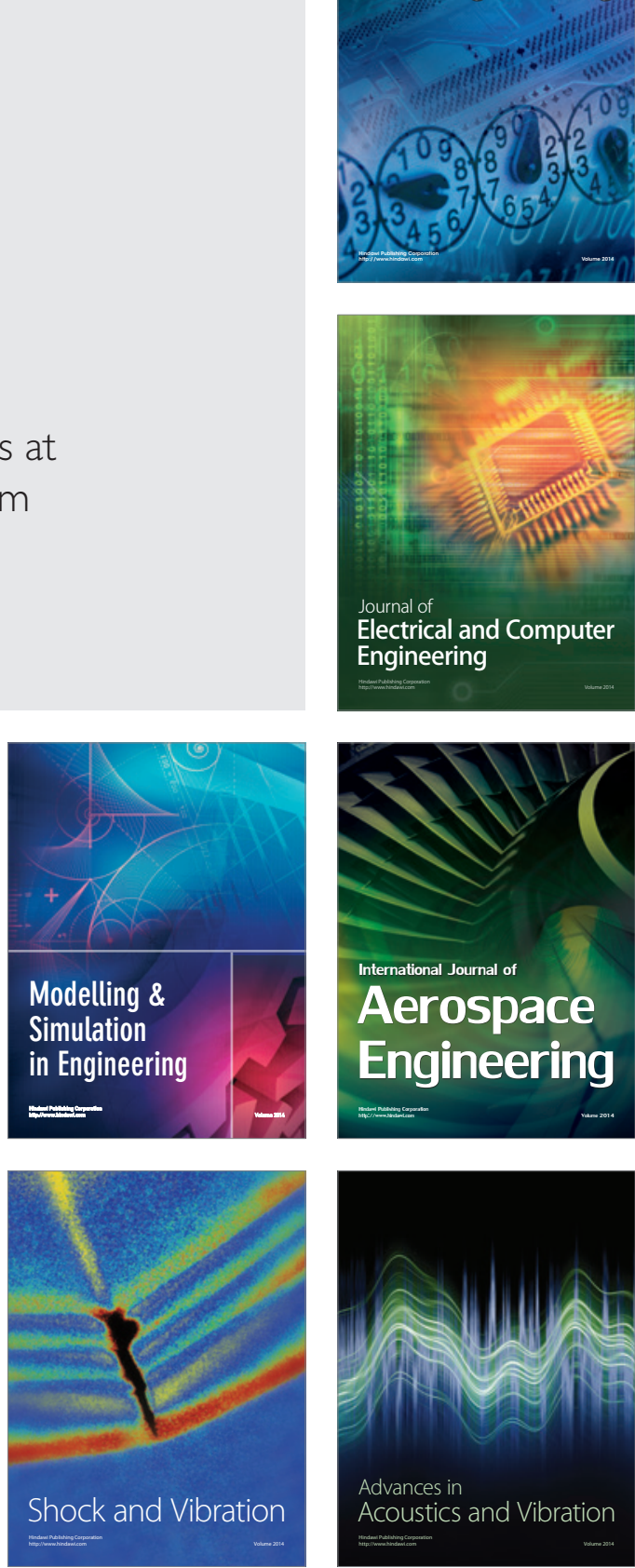